\title{
On the cuspidalization problem for hyperbolic curves over finite fields
}

\author{
Yasuhiro Wakabayashi
}

\begin{abstract}
In this article, we study some group-theoretic constructions associated to arithmetic fundamental groups of hyperbolic curves over finite fields. One of the main results of this article asserts that any Frobenius-preserving isomorphism between the geometrically pro- $l$ fundamental groups of hyperbolic curves with one given point removed induces an isomorphism between the geometrically pro- $l$ fundamental groups of the hyperbolic curves obtained by removing other points. Finally, we apply this result to obtain results concerning certain cuspidalization problems for fundamental groups of (not necessarily proper) hyperbolic curves over finite fields.
\end{abstract}

\section{Introduction}

In the present article, we consider the following problem.

PROBLEM

Suppose that we are given a hyperbolic curve over a finite field in which $l$ is invertible. Then, given the geometrically pro- $l$ fundamental group of the curve obtained by removing a specific point from this hyperbolic curve, is it possible to reconstruct the geometrically pro- $l$ fundamental groups of the curves obtained by removing other points which vary "continuously" in a suitable sense?
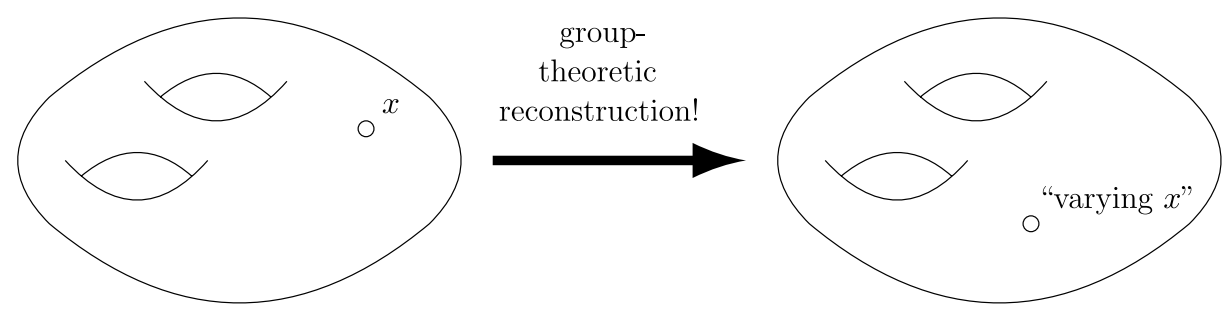

We shall formulate the above problem mathematically.

Let $l$ be a prime number, and let $X$ be a hyperbolic curve over a finite field $K$ in which $l$ is invertible. For $n$ a positive integer, we denote by $X_{n}$ the $n$th configuration space associated to $X$ (hence, $X_{1}=X$ ), and write $\Pi_{X_{n}}$ for the geometrically pro-l fundamental group of $X_{n}$. In the case $n=2$, the fiber of a

Kyoto Journal of Mathematics, Vol. 56, No. 1 (2016), 125-164

DOI 10.1215/21562261-3445174, (C) 2016 by Kyoto University

Received June 26, 2012. Revised April 3, 2014. Accepted December 24, 2014.

2010 Mathematics Subject Classification: Primary 14H30; Secondary 14H10. 
projection $X_{2} \rightarrow X$ over a $K$-rational point $x \in X$ may be naturally identified with $X \backslash\{x\}$, so we may regard $X_{2} \rightarrow X$ as a continuous family of cuspidalizations of $X$. Therefore, the above problem can be formulated as follows (where $Y$ denotes a hyperbolic curve over a finite field $L$ in which $l$ is also invertible, and we use notation for $Y$ that is similar to that for $X$ ).

\section{THEOREM A}

Let

$$
\alpha: \Pi_{X \backslash\{x\}} \stackrel{\sim}{\longrightarrow} \Pi_{Y \backslash\{y\}}
$$

be a Frobenius-preserving isomorphism (see Definition 5.7(a)) which maps a specific decomposition group $D_{x}$ of $x$ onto a specific decomposition group $D_{y}$ of $y$. Here, we shall denote by $\bar{\alpha}: \Pi_{X} \stackrel{\sim}{\rightarrow} \Pi_{Y}$ (resp. $, \bar{D}_{x}, \bar{D}_{y}$ ) the isomorphism (resp., the image of $D_{x}$ in $\Pi_{X}$, the image of $D_{y}$ in $\Pi_{Y}$ ) obtained by passing to the quotients $\Pi_{X \backslash\{x\}} \rightarrow \Pi_{X}$ (resp., $\Pi_{Y \backslash\{y\}} \rightarrow \Pi_{Y}$ ). Then there exists an isomorphism

$$
\alpha_{2}: \Pi_{X_{2}} \stackrel{\sim}{\longrightarrow} \Pi_{Y_{2}}
$$

which is uniquely determined up to composition with an inner automorphism (of either the domain or codomain) by the condition that it is compatible with the natural switching automorphisms up to an inner automorphism (of either the domain or codomain) and fits into a commutative diagram

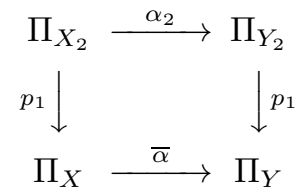

that induces $\alpha$ by restricting $\alpha_{2}$ to the inverse images (via the vertical arrows) of $\bar{D}_{x}$ and $\bar{D}_{y}$. In particular, if $x^{\prime}$ (resp., $\left.y^{\prime}\right)$ is a K-rational point of $X$ (resp., an L-rational point of $Y$ ) and we assume that the decomposition groups of $x^{\prime}, y^{\prime}$ correspond via $\alpha$, then we have an isomorphism

$$
\alpha^{\prime}: \Pi_{X \backslash\left\{x^{\prime}\right\}} \stackrel{\sim}{\longrightarrow} \Pi_{Y \backslash\left\{y^{\prime}\right\}}
$$

(which may not be unique) such that $\alpha$ and $\alpha^{\prime}$ induce the same isomorphism $\Pi_{X} \stackrel{\sim}{\rightarrow} \Pi_{Y}$.

Now let us explain the content of each section briefly. In Section 3, we recall the notion of the (log) configuration space associated to a hyperbolic curve and review group-theoretic properties of the various fundamental groups associated to such spaces. In particular, the splitting determined by the Frobenius action on the pro-l étale fundamental group $\Delta_{X_{n}}$ of $X_{n} \times_{K} \bar{K}$ gives rise to an explicit description of the graded Lie algebra obtained by considering the weight filtration on $\Delta_{X_{n}}$ (see Definition 3.6). This explicit description will play an essential role in the proof of Theorem A.

In Section 4, we discuss a certain specific choice (among composites with inner automorphisms) of the morphism between geometrically pro- $l$ fundamental 
groups obtained by switching the two ordered marked points parameterized by the second configuration space. This choice will play a key role in the proof of Theorem A.

Section 5 is devoted to proving Theorem A. Roughly speaking, starting from a given geometrically pro- $l$ fundamental group $\Pi_{X \backslash\{x\}}$, we reconstruct grouptheoretically a suitable topological group, that is, $\Pi_{X_{2}}^{\mathrm{Lie}}$ (see Definition 5.1), which contains the geometrically pro- $l$ fundamental group of the second configuration space, by using the explicit description of graded Lie algebra studied in Section 3.

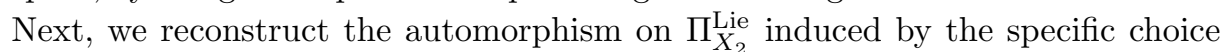
of the switching morphism studied in Section 4. Finally, we verify that $\Pi_{X_{2}}$ can be generated, as a subgroup of $\Pi_{X_{2}}^{\mathrm{Lie}}$, by the given fundamental group $\Pi_{X \backslash\{x\}}$ and the image of this fundamental group via the specific choice of the switching morphism studied in Section 4; this allows us to reconstruct $\Pi_{X_{2}}$ as a subgroup of $\Pi_{X_{2}}^{\mathrm{Lie}}$.

In Section 6, as an application of (a slightly generalized version of) Theorem A, we give a group-theoretic construction of the cuspidalization of an affine hyperbolic curve $X$ over a finite field at a point infinitesimally close to the cusp $x$. That is to say, we give a construction, starting from the geometrically pro- $l$ fundamental group $\Pi_{X}$ of $X$, of the geometrically pro- $l$ fundamental group $\Pi_{\bar{X}_{x}^{\log }}$ of the log scheme obtained by gluing $X$ to a tripod (i.e., the projective line minus three points) at a cusp $x$ of $X$.

\section{THEOREM B}

Let $X$ (resp., $Y$ ) be an affine hyperbolic curve over a finite field $K$ (resp., L), and let $x$ be a $K$-rational point of $\bar{X} \backslash X$ (resp., $y$ an L-rational point of $\bar{Y} \backslash Y$ ). Let

$$
\alpha: \Pi_{X} \stackrel{\sim}{\longrightarrow} \Pi_{Y}
$$

be a Frobenius-preserving isomorphism such that the decomposition groups of $x$ and $y$ (which are well defined up to conjugacy) correspond via $\alpha$. Then there exists an isomorphism

$$
\alpha_{x, y}: \Pi_{\bar{X}_{x}^{\log }} \stackrel{\sim}{\longrightarrow} \Pi_{\bar{Y}_{y}^{\log }}
$$

which is uniquely determined up to composition with an inner automorphism (of either the domain or codomain) by the condition that it maps the conjugacy class of the decomposition group of $\tilde{x}$ to the conjugacy class of the decomposition group of $\tilde{y}$ and induces $\alpha$ upon passing to the quotients $\Pi_{\bar{X}_{x}^{\log }} \rightarrow \Pi_{X}, \Pi_{\bar{Y}_{y}^{\log }} \rightarrow \Pi_{Y}$.

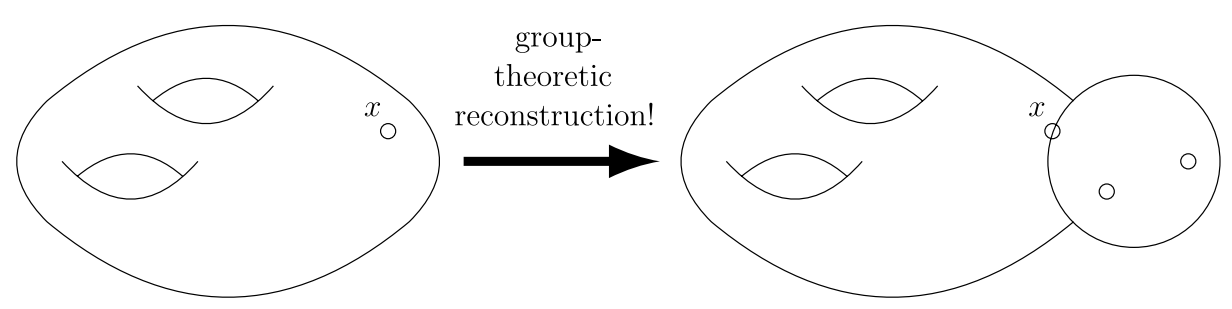


At the end of this article, we consider the cuspidalization problem for (geometrically pro- $l$ ) fundamental groups of configuration spaces of (not necessarily proper) hyperbolic curves over finite fields (see Theorem 6.4).

\section{THEOREM C}

Let $X$ (resp., $Y$ ) be a hyperbolic curve over a finite field $K$ (resp., L). Let

$$
\alpha_{1}: \Pi_{X} \stackrel{\sim}{\longrightarrow} \Pi_{Y}
$$

be a Frobenius-preserving isomorphism. Then for any $n \in \mathbb{Z}_{\geq 0}$, there exists an isomorphism

$$
\alpha_{n}: \Pi_{X_{n}} \stackrel{\sim}{\longrightarrow} \Pi_{Y_{n}}
$$

which is uniquely determined up to composition with an inner automorphism (of either the domain or codomain) by the condition that it is compatible with the natural respective outer actions of the symmetric group on $n$ letters and makes the diagram $(i=1, \ldots, n+1)$

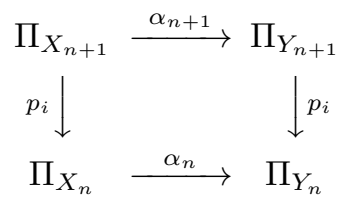

commute.

Finally, we make a remark on the results in the present article. When the curves involved are of genus at least 2, Theorem A may be obtained as an immediate consequence of [12, Theorem 3.1] and [2, Theorem 4.1 and Corollary 4.1(i)]. Also, Theorem $\mathrm{C}$ is already proved in [12] for the case where $n=2$ and $X$ is proper and in [2] for the case where $n \geq 3$ and $X$ is proper. On the other hand, the proof of Theorem A given in the present article is considerably simpler and more direct than the proofs in [12] and [2]. Indeed, in the present article, we shall apply Theorem A to give (see Theorem C) a substantially simpler proof of $[2$, Theorem 4.1] than that given in [2], which, moreover, includes the affine case for the first time.

\section{Notations and conventions}

Numbers. We shall denote by $\mathbb{Q}$ the field of rational numbers, by $\mathbb{Z}$ the ring of rational integers, and by $\mathbb{N} \subseteq \mathbb{Z}$ (resp., $\mathbb{Z}_{\geq a} \subseteq \mathbb{Z}$ ) the additive submonoid of integers $n \geq 0$ (resp., the subset of integers $n \geq a$ for $a \in \mathbb{Z}$ ). If $l$ is a prime number, then $\mathbb{Z}_{l}$ (resp., $\mathbb{Q}_{l}$ ) denotes the l-adic completion of $\mathbb{Z}$ (resp., $\mathbb{Q}$ ).

Topological groups. For an arbitrary Hausdorff topological group $G$, the notation

$$
G^{\mathrm{ab}}
$$


will be used to denote the abelianization of $G$, that is, the quotient of $G$ by the closed subgroup of $G$ topologically generated by the commutators of $G$. We shall say that $G$ is slim if each open subgroup of $G$ is center-free.

For each closed subgroup $H$ of $G$, let us write

$$
N_{G}(H):=\left\{g \in G \mid g \cdot H \cdot g^{-1}=H\right\}
$$

for the normalizer of $H$ in $G$. We shall say that a closed subgroup $H \subseteq G$ is normally terminal in $G$ if the normalizer $N_{G}(H)$ is equal to $H$.

We shall write $\operatorname{Aut}(G)$ for the group of automorphisms of the topological group $G$, Inn : $G \rightarrow \operatorname{Aut}(G)$ for the homomorphism obtained by letting $G$ act on $G$ by inner automorphisms, and $\operatorname{Out}(G):=\operatorname{Aut}(G) / \operatorname{Inn}(G)$.

If $G^{\prime}$ is a topological group, then one may define an equivalence relation $\sim$ on $\operatorname{Hom}\left(G^{\prime}, G\right)$, the set of continuous group homomorphisms from $G^{\prime}$ to $G$, by

$$
f_{1} \sim f_{2} \Longleftrightarrow \exists g \in G: f_{1}=\operatorname{Inn}(g) \circ f_{2},
$$

where $f_{1}, f_{2} \in \operatorname{Hom}\left(G^{\prime}, G\right)$. We shall refer to an element of the quotient set $\operatorname{Hom}\left(G^{\prime}, G\right) / \sim$ of $\operatorname{Hom}\left(G^{\prime}, G\right)$ by $\sim$ as an outer homomorphism. Note that $\sim$ is compatible with composition of homomorphisms, so composition of outer homomorphisms make sense.

If $G$ is center-free, then the natural sequence

$$
1 \longrightarrow G \stackrel{\operatorname{Inn}}{\longrightarrow} \operatorname{Aut}(G) \longrightarrow \operatorname{Out}(G) \longrightarrow 1
$$

is evidently exact. If the profinite group $G$ is topologically finitely generated, then the groups $\operatorname{Aut}(G)$ and $\operatorname{Out}(G)$ are naturally endowed with a profinite topology, and the above sequence may be regarded as an exact sequence of profinite groups.

If, moreover, $J \rightarrow \operatorname{Out}(G)$ is a homomorphism of groups, then we shall write

$$
G \stackrel{\text { out }}{\rtimes}:=\operatorname{Aut}(G) \times \operatorname{Out}(G) J
$$

for the outer semidirect product of $J$ with $G$. Thus, we have a natural exact sequence

$$
1 \longrightarrow G \longrightarrow G \stackrel{\text { out }}{\rtimes} J \longrightarrow J \longrightarrow 1 \text {. }
$$

It has been shown (see [2, Lemma 4.10]) that if an automorphism $\phi$ of $G \stackrel{\text { out }}{\rtimes} J$ preserves the subgroup $G \subseteq G \stackrel{\text { out }}{\rtimes} J$ and induces the identity morphism on $G$ and the quotient $J$, then $\phi$ is the identity morphism of $G \stackrel{\text { out }}{\rtimes} J$.

Log schemes. Basic references for the notion of log scheme are [7] and [6]. In this article, $\log$ structures are always considered on the étale sites of schemes. For a log scheme $X^{\log }$, we shall denote by $X$ (resp., $\mathcal{M}_{X}$ ) the underlying scheme of $X^{\log }$ (resp., the sheaf of monoids defining the log structure of $X^{\log }$ ). Let $X^{\log }$ and $Y^{\log }$ be $\log$ schemes, and let $f^{\log }: X^{\log } \rightarrow Y^{\log }$ be a morphism of $\log$ schemes. Then we shall refer to the quotient of $\mathcal{M}_{X}$ by the image of the morphism $f^{*} \mathcal{M}_{Y} \rightarrow \mathcal{M}_{X}$ induced by $f^{\log }$ as the relative characteristic sheaf of $f^{\log }$. Moreover, we shall refer to the relative characteristic sheaf of the morphism $X^{\log } \rightarrow X$ (where, by abuse of notation, we write $X$ for the log scheme obtained 
by equipping $X$ with the trivial log structure) induced by the natural inclusion $\mathcal{O}^{*} \hookrightarrow \mathcal{M}_{X}$ as the characteristic sheaf of $X^{\log }$.

We shall say that a log scheme $X^{\log }$ is $f s$ if $\mathcal{M}_{X}$ is a sheaf of integral monoids (see [6, Section 1.1]) and, locally for the étale topology, has a chart modeled on a finitely generated and saturated monoid (see [6, Section 1.1]). If $X^{\log }$ is $f s$, then for $n$ a nonnegative integer, we shall refer to the $n$-interior of $X^{\log }$ as the open subset of $X$ on which the associated sheaf of group envelopes (see [6, Section 1.1]) of characteristic sheaf of $X^{\log }$ is of rank $\leq n$. Thus, the 0 -interior of $X^{\log }$ is often referred to simply as the interior of $X^{\log }$.

Curves. Let $f: X \rightarrow S$ be a morphism of schemes. Then we shall say that $f$ is a family of curves of type $(g, r)$ if it factors $X \hookrightarrow \bar{X} \rightarrow S$ as the composite of an open immersion $X \hookrightarrow \bar{X}$ whose image is the complement $\bar{X} \backslash D$ of a relative divisor $D \subseteq \bar{X}$ which is finite étale over $S$ of relative degree $r$ and a morphism $\bar{X} \rightarrow S$ which is proper, smooth, and geometrically connected and whose geometric fibers are one-dimensional of genus $g$. We shall refer to $\bar{X}$ as the compactification of $X$. We shall say that $f$ is a family of hyperbolic curves (resp., tripod) if $f$ is a family of curves of type $(g, r)$ such that $(g, r)$ satisfies $2 g-2+r>0$ (resp., $(g, r)=(0,3)$ and the relative divisor $D$ is split over $S$ ).

We shall denote by

$$
\overline{\mathcal{M}}_{g,[r]+s}
$$

the moduli stack of $(r+s)$-pointed stable curves of genus $g$ for which $s$ sections are equipped with an ordering. This moduli stack may be obtained as the quotient of the moduli stack of ordered $(r+s)$-pointed stable curves of genus $g$ (see [8] for an exposition of the theory of such curves) by a suitable symmetric group action on $r$ letters. We shall denote by $\overline{\mathcal{M}}_{g,[r]+s}^{\log }$ the log stack obtained by equipping $\overline{\mathcal{M}}_{g,[r]+s}$ with the $\log$ structure associated to the divisor with normal crossings which parameterizes singular curves.

Fundamental groups. A basic reference for the notion of Kummer étale covering is [6]. For a locally Noetherian, connected scheme $X$ (resp., locally Noetherian, connected, fs $\log$ scheme $X^{\log }$ ) equipped with a geometric point $\bar{x} \rightarrow X$ (resp., log geometric point $\tilde{x}^{\log } \rightarrow X^{\log }$ ), we shall denote by $\pi_{1}(X, \bar{x})$ (resp., $\left.\pi_{1}\left(X^{\log }, \tilde{x}^{\log }\right)\right)$ the étale fundamental group of $X$ (resp., logarithmic fundamental group of $X^{\log }$; see [1, Chapter 5 , Section 7$]$ ). Since one knows that the étale and logarithmic fundamental groups are determined up to inner automorphisms independently of the choice of base point, we shall omit the base point, and write $\pi_{1}(X)$ (resp., $\left.\pi_{1}\left(X^{\log }\right)\right)$.

For a scheme $X$ (resp., fs log scheme $X^{\log }$ ) which is geometrically connected and of finite type over a field $K$ in which a prime number $l$ is invertible, we shall refer to the quotient $\Pi_{X}$ of $\pi_{1}(X)$ (resp., quotient $\Pi_{X^{\log }}$ of $\pi_{1}\left(X^{\log }\right)$ ) by the closed normal subgroup obtained as the kernel of the natural projection from $\pi_{1}\left(X \times_{K} \bar{K}\right)$ (resp., $\pi_{1}\left(X^{\log } \times_{K} \bar{K}\right)$ ) (where $\bar{K}$ is a separable closure of $K)$ to its maximal pro-l quotient $\Delta_{X}$ (resp., $\Delta_{X^{\log }}$ ) as the geometrically pro-l étale fundamental group of $X$ (resp., geometrically pro-l logarithmic fundamental 
group of $X^{\log }$ ). Thus (if we write $G_{K}$ for the Galois group of a separable closure of $K$ over $K$, then) we have a natural exact sequence

$$
\begin{gathered}
1 \longrightarrow \Delta_{X} \longrightarrow \Pi_{X} \longrightarrow G_{K} \longrightarrow 1 \\
\text { (resp., } 1 \longrightarrow \Delta_{X^{\log }} \longrightarrow \Pi_{X^{\log }} \longrightarrow G_{K} \longrightarrow 1 \text { ). }
\end{gathered}
$$

Note that if the log structure of $X^{\log }$ is trivial, then we have natural isomorphisms $\Delta_{X} \log \stackrel{\sim}{\rightarrow} \Delta_{X}, \Pi_{X^{\log } \stackrel{\sim}{\rightarrow}} \Pi_{X}$.

If $K$ is finite, then write $G_{K}^{\dagger} \subseteq G_{K}$ for the (unique) maximal pro-l subgroup of $G_{K}$ (so $\left.G_{K}^{\dagger} \cong \mathbb{Z}_{l}\right)$. Also, for a profinite group $\Pi$ over $G_{K}$, we shall use the notation

$$
\Pi^{\dagger}:=\Pi \times_{G_{K}} G_{K}^{\dagger} \subseteq \Pi
$$

and refer to it as the restricted pro-l group of $\Pi$.

\section{Fundamental groups of $(\log )$ configuration spaces}

The purpose of this section is to recall the notion of the (log) configuration space associated to a curve and review group-theoretic properties of the various fundamental groups associated to such spaces. Let $l$ be a prime number, let $K$ be a finite field in which $l$ is invertible, let $\bar{K}$ be a separable closure of $K$, where we shall denote by $G_{K}$ the Galois group of $\bar{K}$ over $K$, and let $X$ be a hyperbolic curve over $K$ of type $(g, r)$.

\section{DEFINITION 3.1}

(a) For $n \in \mathbb{Z}_{\geq 1}$, write $X^{\times n}$ for the fiber product of $n$ copies of $X$ over $K$. We shall denote by

$$
X_{n}\left(\subseteq X^{\times n}\right)
$$

the $n$th configuration space associated to $X$, that is, the scheme which represents the open subfunctor

$$
S \mapsto\left\{\left(f_{1}, \ldots, f_{n}\right) \in X^{\times n}(S) \mid f_{i} \neq f_{j} \text { if } i \neq j\right\}
$$

of the functor represented by $X^{\times n}$.

(b) Let us denote by $\bar{X}_{n}^{\log }$ the $n$th $\log$ configuration space associated to $X$ (see [15]); that is,

$$
\bar{X}_{n}^{\log }:=\operatorname{Spec} K \times \overline{\mathcal{M}}_{g,[r]}^{\log } \overline{\mathcal{M}}_{g,[r]+n}^{\log },
$$

where the (1-)morphism Spec $K \rightarrow \overline{\mathcal{M}}_{g,[r]}^{\log }$ is the classifying morphism determined by the curve $X \rightarrow \operatorname{Spec} K$, and the (1-)morphism $\overline{\mathcal{M}}_{g,[r]+n}^{\log } \rightarrow \overline{\mathcal{M}}_{g,[r]}^{\log }$ is obtained by forgetting the ordered $n$ marked points of the tautological family of curves over $\overline{\mathcal{M}}_{g,[r]+n}^{\log }$. In the following, for simplicity, we shall write $\bar{X}^{\log }$ for $\bar{X}_{1}^{\log }$.

\section{PROPOSITION 3.2}

(a) The 0-interior (see the Introduction) of the log scheme $\bar{X}_{n}^{\log }$ is naturally isomorphic to the $n$th configuration space $X_{n}$ associated to $X$. 
(b) The log scheme $\bar{X}_{n}^{\log }$ is log regular and its underlying scheme is connected and regular.

(c) The projection $p_{k}^{\log :} \bar{X}_{n}^{\log } \rightarrow \bar{X}_{n-1}^{\log }$, induced from the (1-)morphism $\overline{\mathcal{M}}_{g,[r]+n}^{\log } \rightarrow \overline{\mathcal{M}}_{g,[r]+n-1}^{\log }$ obtained by forgetting the $k$ th $(k=1, \ldots, n)$ ordered points of the tautological family of curves over $\overline{\mathcal{M}}_{g,[r]+n}^{\log }$, is log smooth (see the Introduction). Its underlying morphism of schemes is the natural projection $p_{k}: X_{n} \rightarrow X_{n-1}$ obtained by forgetting the kth factor and, hence, is flat and has connected and reduced fibers over the geometric points of $X_{n-1}$.

Proof

See, for example, [2, Proposition 2.2].

\section{DEFINITION 3.3}

We shall denote (see the Introduction) by

$$
\Pi_{X_{n}} \quad\left(\text { resp., } \Delta_{X_{n}}\right)
$$

the geometrically pro- $l$ étale fundamental group of $X_{n}$ (resp., $X_{n} \times_{K} \bar{K}$ ) and by

$$
\Pi_{\bar{X}_{n}^{\log }} \quad \text { (resp., } \Pi_{\bar{X}^{\log \times n}} \text { ) }
$$

the geometrically pro- $l \log$ fundamental group of $\bar{X}_{n}^{\log }$ (resp., the fiber product $\bar{X}^{\log \times n}$ of $n$ copies of $\bar{X}^{\log }$ over $K$ ). Moreover, we shall denote (see the Introduction) by

$$
\Pi_{X_{n}}^{\dagger}, \quad \Delta_{X_{n}}^{\dagger}\left(\cong \Delta_{X_{n}}\right), \quad \Pi_{\bar{X}_{n}^{\log }}^{\dagger}, \quad \Pi_{\bar{X}^{\log \times n}}^{\dagger}
$$

the respective restricted geometrically pro- $l$ groups.

Also we shall write

$$
p_{k}^{\Delta}: \Delta_{X_{n}} \rightarrow \Delta_{X_{n-1}}, \quad p_{k}^{\Pi}: \Pi_{X_{n}} \rightarrow \Pi_{X_{n-1}}
$$

for the morphisms induced by the projection $p_{k} \times_{K} \bar{K}: X_{n} \times_{K} \bar{K} \rightarrow X_{n-1} \times_{K}$ $\bar{K}, p_{k}: X_{n} \rightarrow X_{n-1}$ obtained by forgetting the $k$ th factor (these morphisms of profinite groups are only defined up to conjugacy in the absence of appropriate choices of base points of respective schemes), and write

$$
i_{k}^{\Delta}: \Delta_{X_{n / n-1}}^{k} \hookrightarrow \Delta_{X_{n}}, \quad i_{k}^{\Delta^{\prime}}: \Delta_{X_{n / n-1}}^{k} \hookrightarrow \Pi_{X_{n}}
$$

for the kernels of the surjections $p_{k}^{\Delta}: \Delta_{X_{n}} \rightarrow \Delta_{X_{n-1}}, p_{k}^{\Pi}: \Pi_{X_{n}} \rightarrow \Pi_{X_{n-1}}$. Then we have the exact sequences

$$
\begin{aligned}
& 1 \longrightarrow \Delta_{X_{n}} \longrightarrow \Pi_{X_{n}}^{(-)} \longrightarrow G_{K}^{(-)} \longrightarrow 1, \\
& 1 \longrightarrow \Delta_{X_{n / n-1}}^{k} \stackrel{i_{k}^{\Delta}}{\longrightarrow} \Delta_{X_{n}} \stackrel{p_{k}^{\Delta}}{\longrightarrow} \Delta_{X_{n-1}} \longrightarrow 1, \\
& 1 \longrightarrow \Delta_{X_{n / n-1}}^{k} \stackrel{i_{k}^{\Delta^{\prime}}}{\longrightarrow} \Pi_{X_{n}}^{(-)} \stackrel{p_{k}^{\Pi^{(-)}}}{\longrightarrow} \Pi_{X_{n-1}}^{(-)} \longrightarrow 1,
\end{aligned}
$$

where the symbol $(-)$ denotes either the presence or absence of $\dagger$. 
Also, we have a square diagram

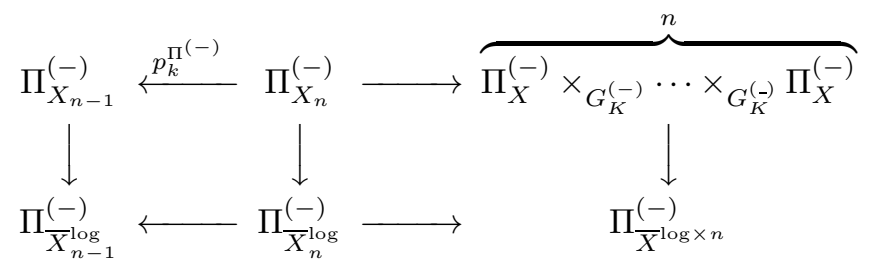

which can be made commutative without conjugate indeterminacy by choosing compatible base points, arising from a natural commutative diagram

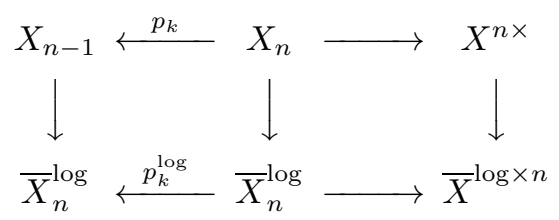

Then, it follows from Propositions 3.2(a) and 3.2(b) together with the log purity theorem (see [6], [9]) that the three vertical homomorphisms are isomorphisms. In the following, we shall identify $\Pi_{X_{n}}^{(-)}$with $\Pi_{\bar{X}_{n}^{\log }}^{(-)}, \Pi_{\bar{X}^{\log \times n}}^{(-)}$with

$$
\overbrace{\Pi_{X}^{(-)} \times_{G_{K}^{(-)}} \cdots \times_{G_{K}^{(-)}} \Pi_{X}^{(-)}}^{n},
$$

and the surjection $p_{k}^{\Pi}: \Pi_{X_{n}} \rightarrow \Pi_{X_{n-1}}$ with the surjection $\Pi_{\bar{X}_{n}^{\log }}^{(-)} \rightarrow \Pi_{\bar{X}_{n-1}^{\log }}^{(-)}$by means of these specific isomorphisms.

\section{PROPOSITION 3.4}

(a) $\Delta_{X_{n / n-1}}^{k}$ may be naturally identified with the maximal pro-l quotient of the étale fundamental group of a geometric fiber of the projection morphism $p_{k}$ : $X_{n} \rightarrow X_{n-1}$.

(b) The images of the $i_{k}^{\Delta}: \Delta_{X_{n / n-1}}^{k} \rightarrow \Delta_{X_{n}}$, where $k=1, \ldots, n$, generate $\Delta_{X_{n}}$.

(c) The profinite groups $\Delta_{X_{n}}, \Delta_{X_{n / n-1}}^{k}, \Pi_{X_{n}}^{\dagger}$, and $\Pi_{X^{\times n}}^{\dagger}$ are slim (see the Introduction).

\section{Proof}

Assertion (a) follows from [15, Proposition 2.2] or [19, Proposition 2.3]. Assertions (b) and (c) follow from induction on $n$ together with the exact sequence

$$
1 \longrightarrow \Delta_{X_{n / n-1}}^{n} \stackrel{i_{n}^{\Delta}}{\longrightarrow} \Delta_{X_{n}} \stackrel{p_{n}^{\Delta}}{\longrightarrow} \Delta_{X_{n-1}} \longrightarrow 1
$$

displayed in Definition 3.3. Indeed, with regard to (b), $\Delta_{X_{n / n-1}}^{k}$ maps to $\Delta_{X_{n-1 / n-2}}^{k}($ for $k=1, \ldots, n-1)$ via $p_{n}^{\Delta}: \Delta_{X_{n}} \rightarrow \Delta_{X_{n-1}}$, and it is verified that this map $\Delta_{X_{n / n-1}}^{k} \rightarrow \Delta_{X_{n-1 / n-2}}^{k}$ is surjective by regarding it as the morphism 
induced by an open immersion between the hyperbolic curves that arise as geometric fibers of the projection morphisms involved. With regard to (c), the slimness of $\Delta_{X}$ is well known (see, e.g., [10, Lemma 1.3.10]); the slimness of $\Pi_{X}^{\dagger}$ follows from the fact that the character of $G_{K}^{\dagger}$ arising from the determinant of $\Delta_{X}^{\mathrm{ab}}$ coincides with some positive power of the cyclotomic character; the other statements follow from the fact that an extension of slim profinite groups is itself slim.

Next, we recall from [12, Section 3] the theory of the weight filtration of fundamental groups and the associated graded Lie algebra.

\section{DEFINITION 3.5}

Let $l$ be a prime number, let $G, H$, and $A$ be topologically finitely generated pro- $l$ groups, and let $\phi: H \rightarrow A$ be a (continuous) surjective homomorphism. Suppose further that $A$ is abelian, and suppose that $G$ is an $l$-adic Lie group.

(a) We shall use the term central filtration $\{H(n)\}_{n \geq 1}$ on $H$ with respect to the homomorphism $\phi$ for the filtration defined as follows:

$$
\begin{aligned}
H(1) & :=H, \\
H(2) & :=\operatorname{Ker}(\phi), \\
H(m) & :=\left\langle\left[H\left(m_{1}\right), H\left(m_{2}\right)\right] \mid m_{1}+m_{2}=m\right\rangle \text { for } m \geq 3,
\end{aligned}
$$

where $\left\langle N_{i} \mid i \in I\right\rangle$ is the group topologically generated by the $N_{i}$ 's.

In the following, for $a, b, n \in \mathbb{Z}$ such that $1 \leq a \leq b, n \geq 1$, we shall write

$$
\begin{aligned}
H(a / b) & :=H(a) / H(b), \\
\operatorname{Gr}(H) & :=\bigoplus_{m \geq 1} H(m / m+1), \\
\operatorname{Gr}(H)(a / b) & :=\bigoplus_{b>m \geq a} H(m / m+1), \\
\operatorname{Gr}_{\mathbb{Q}_{l}}(H) & :=\operatorname{Gr}(H) \otimes_{\mathbb{Z}_{l}} \mathbb{Q}_{l}, \\
\operatorname{Gr}_{\mathbb{Q}_{l}}(a / b) & :=\operatorname{Gr}(H)(a / b) \otimes_{\mathbb{Z}_{l}} \mathbb{Q}_{l}, \\
H(a / \infty) & :={\underset{l}{b>a}}_{\leftarrow} H(a / b) .
\end{aligned}
$$

(b) We shall denote by $\operatorname{Lie}(G)$ the Lie algebra over $\mathbb{Q}_{l}$ determined by the $l$-adic Lie group $G$. We shall say that $G$ is nilpotent if there exists a positive integer $m$ such that if we denote by $\{G(n)\}$ the central filtration with respect to the natural surjection $G \rightarrow G^{\text {ab }}$ (see (a)), then $G(m)=\{1\}$. If $G$ is nilpotent, then $\operatorname{Lie}(G)$ is a nilpotent Lie algebra over $\mathbb{Q}_{l}$ and, hence, determines a connected, unipotent linear algebraic group $\operatorname{Lin}(G)$, which we shall refer to as the linear algebraic group associated to $G$. In this situation, there exists a natural 
(continuous) homomorphism (with open image)

$$
G \longrightarrow \operatorname{Lin}(G)\left(\mathbb{Q}_{l}\right)
$$

(from $G$ to the $l$-adic Lie group determined by the $\mathbb{Q}_{l}$-valued points of $\operatorname{Lin}(G)$ ) which is uniquely determined ( $\operatorname{since} \operatorname{Lin}(G)$ is connected and unipotent) by the condition that it induces the identity morphism on the associated Lie algebras. In the situation of (a), if $1 \leq a \in \mathbb{Z}$, then we shall write

$$
\begin{aligned}
& \operatorname{Lie}(H(a / \infty)):=\lim _{b>a} \operatorname{Lie}(H(a / b)), \\
& \operatorname{Lin}(H(a / \infty)):=\underbrace{}_{b>a} \operatorname{Lin}(H(a / b)),
\end{aligned}
$$

where we note that each $H(a / b)$ is a nilpotent $l$-adic Lie group.

\section{DEFINITION 3.6}

For $n \in \mathbb{Z}_{\geq 1}$, we shall denote by

$$
\left\{\Delta_{X_{n}}(m)\right\}
$$

the central filtration of $\Delta_{X_{n}}$ with respect to the natural surjection $\Delta_{X_{n}} \rightarrow \Delta_{\bar{X} \times n}^{\mathrm{ab}}$ (where $\bar{X}$ denotes the smooth compactification of $X$ (see the Introduction)), and refer to it as the weight filtration on $\Delta_{X_{n}}$.

\section{PROPOSITION 3.7}

If we equip $\Delta_{X_{n / n-1}}^{k}$ with the central filtration induced from the identification given by Proposition 3.4(a) and its weight filtration, then the sequence of morphisms of graded Lie algebras

$$
1 \longrightarrow \operatorname{Gr}\left(\Delta_{X_{n / n-1}}^{k}\right) \stackrel{\operatorname{Gr}\left(i_{k}^{\Delta}\right)}{\longrightarrow} \operatorname{Gr}\left(\Delta_{X_{n}}\right) \stackrel{\operatorname{Gr}\left(p_{k}^{\Delta}\right)}{\longrightarrow} \operatorname{Gr}\left(\Delta_{X_{n-1}}\right) \longrightarrow 1
$$

induced by the second displayed exact sequence of Definition 3.3 is exact.

Proof

See [2, Proposition 4.1].

Next, let us fix a section $\sigma: G_{K} \rightarrow \Pi_{X_{n}}$ of the surjection $\Pi_{X_{n}} \rightarrow G_{K}$ arising from the structure morphism of $X_{n}$. This section $\sigma$ determines the action of $G_{K}$ on $\Delta_{X_{n}}$ by conjugation, and hence also on

$$
\operatorname{Gr}_{\mathbb{Q}_{l}}\left(\Delta_{X_{n}}\right)(a / b), \quad \operatorname{Lie}\left(\Delta_{X_{n}}(a / b)\right), \quad \operatorname{Lin}\left(\Delta_{X_{n}}(a / b)\right)\left(\mathbb{Q}_{l}\right),
$$

where $a, b \in \mathbb{Z}$ such that $1 \leq a \leq b$.

\section{PROPOSITION 3.8}

Let us assume that $K$ is a finite field whose cardinality we denote by $q_{K}$, and write $\mathrm{Fr} \in G_{K}$ for the Frobenius element of $G_{K}$. Then, relative to the natural conjugate actions determined by $\sigma$, we have the following statements. 
(a) The eigenvalues of the action of $\mathrm{Fr}$ on $\operatorname{Lie}_{X_{n}}(a / a+1)$ are algebraic numbers all of whose complex absolute values are equal to $q_{K}^{a / 2}$ (i.e., weight a).

(b) There is a unique $G_{K}$-equivariant isomorphism of Lie algebras

$$
\operatorname{Lie}\left(\Delta_{X_{n}}(a / b)\right) \stackrel{\sim}{\rightarrow} \operatorname{Gr}_{\mathbb{Q}_{l}}\left(\Delta_{X_{n}}\right)(a / b)
$$

which induces the identity isomorphism

$$
\operatorname{Lie}\left(\Delta_{X_{n}}(c / c+1)\right) \stackrel{\sim}{\rightarrow} \operatorname{Gr}_{\mathbb{Q}_{l}}\left(\Delta_{X_{n}}\right)(c / c+1)
$$

for all $c \in \mathbb{Z}_{\geq 1}$ such that $a \leq c<b$.

Proof

Assertion (a) follows from the "Riemann hypothesis for abelian varieties over finite fields" (see, e.g., [16, p. 206]). Assertion (b) follows formally from assertion (a) by considering the eigenspaces with respect to the action of $\mathrm{Fr}$.

The following proposition is a special case of a result proven previously (see [18]). For simplicity, we discuss only the case used in the proofs of the present article.

PROPOSITION 3.9

For $n=1,2$, the graded Lie algebra $\operatorname{Gr}\left(\Delta_{X_{n}}\right)$ has the following presentation.

(a) The case $n=1$ (i.e., $\left.X_{n}=X\right)$ has generators $(1 \leq j \leq r, 1 \leq i \leq g) \zeta_{j} \in$ $\Delta_{X}(2 / 3)$ and $\alpha_{i}, \beta_{i} \in \Delta_{X}(1 / 2)$ and relation $\sum_{j=1}^{r} \zeta_{j}+\sum_{i=1}^{g}\left[\alpha_{i}, \beta_{i}\right]=0$, where $\zeta_{j}$ $(j=1,2, \ldots, r)$ topologically generates the inertia subgroup in $\Delta_{X}$ (well defined up to conjugacy) associated to the $j$ th cusp (relative to some ordering of the cusps of $\left.X \times_{K} \bar{K}\right)$.

(b) The case $n=2$ has generators $(1 \leq j \leq r, 1 \leq i \leq g, k=1,2) \zeta \in$ $\Delta_{X_{2}}(2 / 3), \zeta_{j}^{k} \in \Delta_{X_{2 / 1}}^{k}(2 / 3)$, and $\alpha_{i}^{k}, \beta_{i}^{k} \in \Delta_{X_{2 / 1}}^{k}(1 / 2)$ and relations $\left(1 \leq j, j^{\prime} \leq r\right.$, $\left.j \neq j^{\prime}, 1 \leq i, i^{\prime} \leq g,\left\{k, k^{\prime}\right\}=\{1,2\}\right) \zeta+\sum_{j=1}^{r} \zeta_{j}^{k}+\sum_{i=1}^{g}\left[\alpha_{i}^{k}, \beta_{i}^{k}\right]=0,\left[\alpha_{i}^{k}, \zeta_{j}^{k^{\prime}}\right]=$ $\left[\beta_{i}^{k}, \zeta_{j}^{k^{\prime}}\right]=0,\left[\zeta_{j}^{k}, \zeta_{j^{\prime}}^{k^{\prime}}\right]=0,\left[\alpha_{i}^{k}, \alpha_{i^{\prime}}^{k^{\prime}}\right]=\left[\beta_{i}^{k}, \beta_{i^{\prime}}^{k^{\prime}}\right]=0$, and

$$
\left[\alpha_{i}^{k}, \beta_{i^{\prime}}^{k^{\prime}}\right]= \begin{cases}\zeta & \text { if } i=i^{\prime}, \\ 0 & \text { if } i \neq i^{\prime},\end{cases}
$$

where $\zeta$ topologically generates the image in $\Delta_{X_{2}}(2 / 3)$ of the inertia subgroup in $\Delta_{X_{2}}$ (well defined up to conjugacy) associated to the diagonal divisor of $X \times_{K}$ $X$, and $\zeta_{j}^{k}$ generates the image in $\Delta_{X_{2 / 1}}^{k}(2 / 3)$ of the inertia subgroup in $\Delta_{X_{2 / 1}}^{k}$ associated to the $j$ th cusp (relative to some ordering of the cusps of $X \times_{K} \bar{K}$ ) of the $k$ th factor of $X_{2}$.

\section{Switching morphism on configuration spaces}

We continue to use the notation of Section 3. In this section, we shall introduce certain closed subschemes of $\bar{X}_{2}^{\log }$ equipped with induced log structures (denoted 
by $\mathbb{D}^{\log }$ and $\bar{X}_{x}^{\log }$ ) and consider various automorphisms induced by the automorphism of $\bar{X}_{2}^{\log }$ determined by switching the two factors of $X$. The geometry of such log schemes allows us to prove the uniqueness of certain specific conjugates of induced switching morphisms between fundamental groups that satisfy certain conditions. This uniqueness (see Proposition 4.5) plays a key role in the proof of Theorem A.

First, we define a log scheme

$$
\mathbb{D}^{\log }
$$

to be the log scheme obtained by equipping the diagonal divisor $\bar{X} \subseteq \bar{X}_{2}$ (which is the restriction of the (1-)morphism $\overline{\mathcal{M}}_{g,[r]+1} \rightarrow \overline{\mathcal{M}}_{g,[r]+2}$ obtained by gluing the tautological family of curves over $\overline{\mathcal{M}}_{g,[r]+1}^{\log }$ to a trivial family of tripods along the final ordered marked section) with the log structure pulled back from $\bar{X}_{2}^{\log }$. Thus, if we write $d: \mathbb{D}^{\log } \rightarrow \bar{X}_{2}^{\log }$ for the natural diagonal embedding, then it follows immediately from the definitions that $p_{1} \circ d=p_{2} \circ d: \mathbb{D}^{\log } \rightarrow \bar{X}^{\log }$ is a morphism of type $\mathbb{N}$ (see [3]), that is, the underlying morphism of schemes is an isomorphism, and the relative characteristic sheaf (see the Introduction) is locally constant with stalk isomorphic to $\mathbb{N}$.

Observe that the (1-)automorphism on $\overline{\mathcal{M}}_{g,[r]+2}^{\log }$ over $\overline{\mathcal{M}}_{g,[r]}^{\log }$ given by switching the two ordered marked points of the tautological family of curves over $\overline{\mathcal{M}}_{g,[r]+2}^{\log }$ induces automorphisms $s, \bar{s}$, and $s_{\mathbb{D}}$, which fit into a commutative diagram as follows:

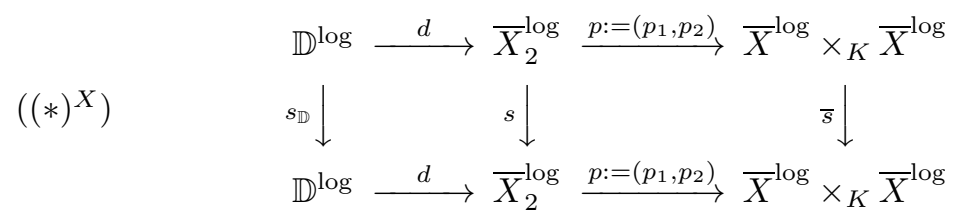

LEMMA 4.1

In the notation of the above situation,

(a) $\bar{s}$ is the morphism determined by switching the two factors; and

(b) $s_{\mathbb{D}}$ is the identity morphism on the underlying scheme.

On the sheaf of monoids defining the log structure of $\mathbb{D}^{\text {log }}$, for any étale local section $s$ of $\mathcal{M}_{\mathbb{D}}$ such that $s=0$ defines the diagonal divisor $\bar{X} \subseteq \bar{X}_{2}$,

$$
s_{\mathbb{D}}(s)=-s .
$$

\section{Proof}

Recall (see [8, Corollary 2.6]) that $\bar{X}_{2}$ is obtained by blowing up $\bar{X} \times_{K} \bar{X}$ along the intersection of the diagonal divisor and the pullbacks of the cusps via $p_{1}, p_{2}: \bar{X}_{2} \rightarrow \bar{X}$. Thus, one verifies easily that assertions (a) and (b) follow immediately from the fact that the ring homomorphism corresponding to $\bar{s}$ in an affine neighborhood of any diagonal point may be expressed as 


$$
\begin{aligned}
A \otimes_{K} A & \longrightarrow A \otimes_{K} A, \\
\sum_{j} a_{j} \otimes a_{j}^{\prime} & \mapsto \sum_{j} a_{j}^{\prime} \otimes a_{j},
\end{aligned}
$$

and hence, it maps $s$ to $-s$ for any local section $s$ such that $s=0$ defines the diagonal divisor $\bar{X} \subseteq \bar{X} \times_{K} \bar{X}$.

\section{REMARK 4.1.1}

Lemma 4.1(b) can be interpreted as the assertion that the automorphism induced by $s_{\mathbb{D}}$ on the sheaf of monoids $\mathcal{M}_{\mathbb{D}}$ defining the log structure of $\mathbb{D}^{\text {log }}$ may be expressed, relative to the étale local splitting of $\mathcal{M}_{\mathbb{D}} \rightarrow \mathcal{M}_{\mathbb{D}} / \mathcal{O}_{X}^{*} \cong \mathbb{N}$ corresponding to $s$, as

$$
\begin{aligned}
\mathbb{N} \oplus \mathcal{O}_{X}^{*} & \stackrel{\sim}{\longrightarrow} \mathbb{N} \oplus \mathcal{O}_{X}^{*}, \\
(m, v) & \longmapsto\left(m,(-1)^{m} v\right) .
\end{aligned}
$$

Next, we introduce the $\log$ scheme $\bar{X}_{x}^{\log }$ that appears in the discussion at the beginning of this section. Let $x^{\log } \rightarrow \bar{X}^{\log }$ be a strict morphism (see [6, Section 1.2]) such that the underlying scheme of $x^{\log }$ is $K$-isomorphic to $\operatorname{Spec}(K)$. We shall write

$$
\begin{aligned}
\bar{X}_{x}^{\log } & :=x^{\log } \times_{X^{\log }} \bar{X}_{2}^{\log }, \\
\tilde{x}^{\log } & :=x^{\log } \times_{\bar{X}^{\log }} \mathbb{D}^{\log },
\end{aligned}
$$

where the morphism $\bar{X}_{2}^{\log } \rightarrow \bar{X}^{\log }$ (resp., $\mathbb{D}^{\log } \rightarrow \bar{X}^{\log }$ ) in the fiber product defining $\bar{X}_{x}^{\log }$ (resp., $\tilde{x}^{\log }$ ) is $p_{1}$ (resp., $p_{1} \circ d=p_{2} \circ d$ ), and we refer to $\bar{X}_{x}^{\log }$ (resp., $\tilde{x}^{\log }$ ) as the cuspidalization of $X$ at $x$ (resp., diagonal cusp of $\bar{X}_{x}^{\log }$ ). We note that both the $\log$ structure of $x^{\log }$ and the underlying scheme of $\bar{X}_{x}^{\log }$ depend on the choice of $x \in \bar{X}$.

The case $x \in X$. In this case, $x=x^{\log }$; that is, the $\log$ structure of $x^{\log }$ is trivial. As we discussed in Section 3, the underlying scheme of $\bar{X}_{x}^{\log }$ is naturally isomorphic to $\bar{X}$; this isomorphism maps $\tilde{x}$ to $x$ and the interior of $\bar{X}_{x}^{\log }$ onto $X \backslash\{x\}$.

The case $x \in \bar{X} \backslash X$. In this case, the log structure of $x^{\log }$ has a chart modeled on $\mathbb{N}$, which determines a local uniformizer of $X$ at $x$. The scheme $\bar{X}_{x}$ consists of precisely two irreducible components, one of which maps to the point $x \in X$ (resp., maps isomorphically to $\bar{X}$ ) via $\bar{X}_{x}^{\log \stackrel{p_{2} \circ i_{1}}{\longrightarrow}} \bar{X}^{\log }$; denote this irreducible component by $\overline{\mathbb{P}}_{K}$ (resp., $\bar{X}$, via a slight abuse of notation). Thus, $\bar{X}$ and $\overline{\mathbb{P}}_{K}$ are joined at a single node $\nu_{x}$. Let us refer to $\bar{X}$ (resp., $\overline{\mathbb{P}}_{K}, \nu_{x}$ ) as the major cuspidal component (resp., the minor cuspidal component, the nexus) at $x$, and denote by $\bar{X}^{\log ^{\prime}}, \overline{\mathbb{P}}_{K}^{\log ^{\prime}}, \nu_{x}^{\log }$ the $\log$ schemes obtained by equipping $\bar{X}, \overline{\mathbb{P}}_{K}, \nu_{x}$ with the respective $\log$ structures pulled back from $\bar{X}_{x}^{\log }$ (see [14, Definition 1.4]). Note that the 1-interior of $\bar{X}^{\log ^{\prime}}$ (resp., $\overline{\mathbb{P}}_{K}^{\log ^{\prime}}$ ) is naturally isomorphic to $X$ (resp., is a tripod). 


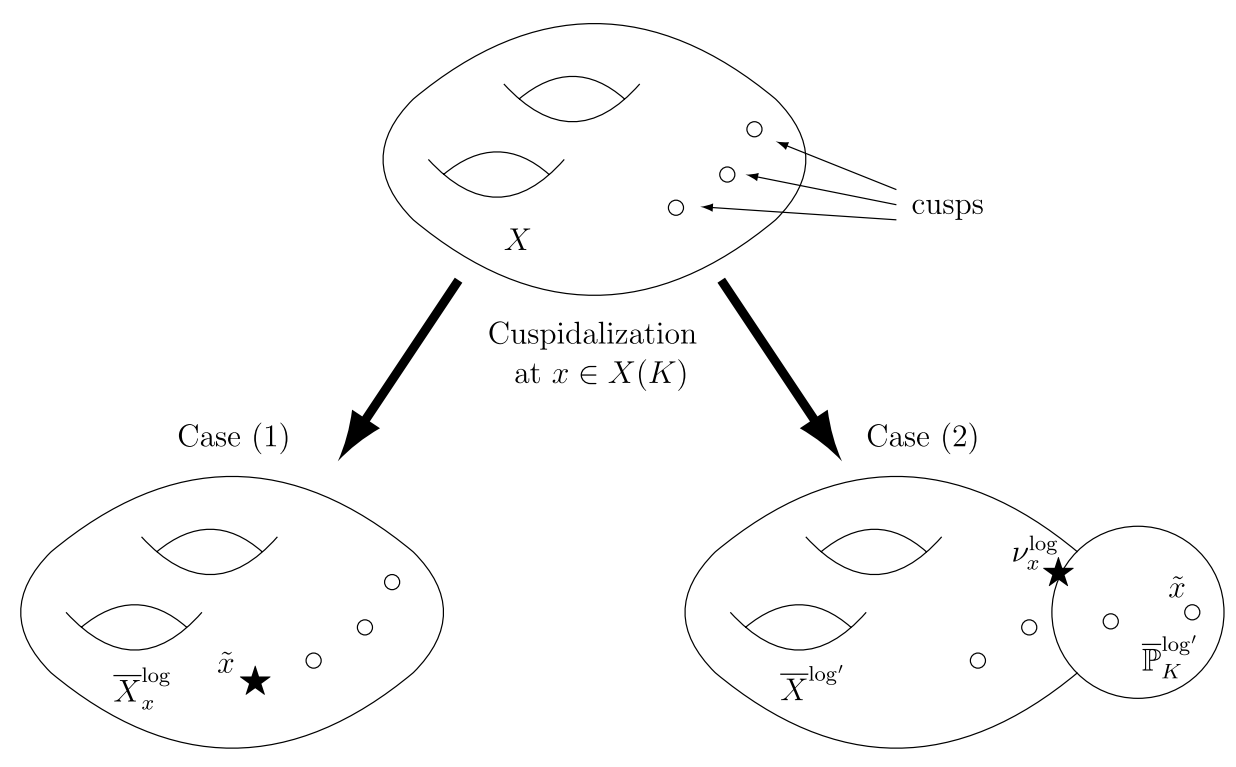

Now, if we denote by

$$
\Pi_{\mathbb{D}^{\log }, \quad \Pi_{\bar{X}_{x}^{\log }}}
$$

the geometrically pro- $l \log$ fundamental groups of $\mathbb{D}^{\log }, \bar{X}_{x}^{\log }$, respectively, then the map $i_{1}: \bar{X}_{x}^{\log } \rightarrow \bar{X}_{2}^{\log }$ of $\log$ schemes induces an outer homomorphism $\left[i_{1}^{\Pi}\right]$ : $\Pi_{\bar{X}_{x}^{\log }} \rightarrow \Pi_{X_{2}}$ of profinite groups (see the Introduction), and the above diagram $\left((*)^{X}\right)$ induces a diagram of outer homomorphisms of profinite groups as follows:

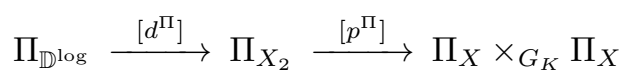

$$
\begin{aligned}
& \left((*)^{\Pi}\right) \quad\left[s_{\mathbb{D}}^{\Pi}\right] \downarrow 2 \quad\left[s^{\Pi}\right] \downarrow 2 \quad\left[\bar{s}^{\Pi}\right] \downarrow \text { 2 }
\end{aligned}
$$

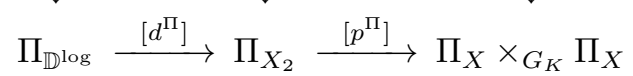

Note that the homomorphisms corresponding to the arrow $\left[i_{1}^{\Pi}\right]$ and the arrows in the diagram $\left((*)^{\Pi}\right)$ are only defined (i.e., in the absence of appropriate choices of base points of respective log schemes) up to conjugacy, and that $\left[\bar{s}^{\Pi}\right]$ coincides with the morphism obtained by switching the two factors. The main purpose of this section is to give characterizations of certain specific choices within these conjugacy classes of homomorphisms.

\section{DEFINITION 4.2}

(a) We shall denote by

$$
\left(C_{i_{1}}^{X}\right) \quad\left(\text { resp., }\left(C_{p_{1}}^{X}\right),\left(C_{p_{2}}^{X}\right),\left(C_{d}^{X}\right)\right)
$$

a choice of a specific homomorphism (i.e., in the sense that it is not subject to conjugacy indeterminacy) 


$$
\begin{gathered}
i_{1}^{\Pi}: \Pi_{\bar{X}_{x}^{\log }} \longrightarrow \Pi_{X_{2}} \\
\text { (resp., } p_{1}^{\Pi}: \Pi_{X_{2}} \longrightarrow \Pi_{X}, p_{2}^{\Pi}: \Pi_{X_{2}} \longrightarrow \Pi_{X}, d^{\Pi}: \Pi_{\mathbb{D}^{\log }} \longrightarrow \Pi_{X_{2}} \text { ) }
\end{gathered}
$$

induced by the morphism of $\log$ schemes $i_{1}: \bar{X}_{x}^{\log } \rightarrow \bar{X}_{2}^{\log }$ (resp., $p_{1}: \bar{X}_{2}^{\log } \rightarrow \bar{X}^{\log }$, $\left.p_{1}: \bar{X}_{2}^{\log } \rightarrow \bar{X}^{\log }, \mathbb{D}^{\log } \rightarrow \bar{X}_{2}^{\log }\right)$.

(b) We shall denote by

$$
\left(C_{\tilde{x}}^{X}\right) \quad\left(\text { resp., }\left(C_{D}^{X}\right)\right)
$$

a choice of a specific subgroup (i.e., of a specific decomposition group)

$$
\left.D_{\tilde{x}} \subseteq \Pi_{\bar{X}_{x}^{\log }} \quad \text { (resp., } D_{X} \subseteq \Pi_{X_{2}}\right)
$$

associated to $\tilde{x}^{\log }$ of $\bar{X}_{x}^{\log }$ (resp., the diagonal divisor of $\bar{X}_{2}$ ), among the various conjugates of this subgroup. Note that such a choice determines a choice of a specific subgroup (i.e., of a specific inertia group)

$$
I_{\tilde{x}}:=D_{\tilde{x}} \cap \Delta_{X_{2 / 1}^{1}} \subseteq \Pi_{\bar{X}_{x}^{\log }} \quad\left(\text { resp., } I_{X}:=D_{X} \cap \Delta_{X_{2 / 1}^{1}} \subseteq \Pi_{X_{2}}\right)
$$

among the various conjugates of this subgroup.

(c) Assume that we have fixed a choice $\left(C_{\tilde{x}}^{X}\right)$ of a specific decomposition group $D_{\tilde{x}} \subseteq \Pi_{\bar{X}_{x}^{\log }}$ (hence also of a specific inertia group $I_{\tilde{x}} \subseteq \Pi_{\bar{X}_{x}^{\log }}$ ). Then we shall denote by

$$
\left(C_{\sigma}^{(-) X}\right)
$$

a choice of a specific section

$$
\sigma^{(-)}: G_{K}^{(-)} \longrightarrow D_{\tilde{x}}^{(-)}
$$

where the symbol (-) denotes either the presence or absence of $\dagger$ (thus, a choice $\left(C_{\sigma}^{X}\right)$ determines a unique choice $\left(C_{\sigma}^{\dagger X}\right)$ by restriction), of the natural surjection $D_{\tilde{x}}^{(-)} \rightarrow G_{K}^{(-)}$(see Remark 4.2.1), and by

$$
\left(C_{\delta}^{X}\right)
$$

a choice of a specific 1-cocycle map

$$
\delta: G_{K}^{\dagger} \longrightarrow I_{\tilde{x}}
$$

representing the Kummer class $-1 \in\left(K^{\times}\right)^{\wedge}$ (see Remark 4.2.1).

Before proceeding, we pause to make a remark concerning Definition 4.2.

\section{REMARK 4.2.1}

(a) Recall that the natural surjection $D_{\tilde{x}} \rightarrow G_{K}$ (which, since $G_{K}$ is abelian, is uniquely determined without any conjugacy indeterminacies) has a section. Indeed, when $x \in X$ (resp., $x \in \bar{X} \backslash X$ ), fixing a choice of such a section is equivalent to extracting roots of any local uniformizer(s) of the $\operatorname{divisor}(\mathrm{s}) \mathbb{D} \subseteq \bar{X}_{2}$ (resp., $\mathbb{D} \subseteq \bar{X}_{2}$ and $\bar{X}_{x} \subseteq \bar{X}_{2}$ ) at $\tilde{x}$. 
(b) We shall consider the restriction map $H^{1}\left(G_{K}, I_{\tilde{x}}\right) \rightarrow H^{1}\left(G_{K}^{\dagger}, I_{\tilde{x}}\right)$ of cohomology groups induced by the natural inclusion $G_{K}^{\dagger} \hookrightarrow G_{K}$. Since $G_{K}^{\dagger}$ is the maximal pro-l subgroup of $G_{K}$ and $I_{\tilde{x}}$ is isomorphic to $\mathbb{Z}_{l}(1)$ as a $G_{K}$-module, this restriction map determines an isomorphism of $H^{1}\left(G_{K}, I_{\tilde{x}}\right)$ with $H^{1}\left(G_{K}^{\dagger}, I_{\tilde{x}}\right)$ and, hence, also with the maximal pro- $l$ completion $\left(K^{\times}\right)^{\wedge}$ of the multiplicative group $K^{\times}$of $K$. Therefore, if we denote by $Z^{1}\left(G_{K}^{\dagger}, I_{\tilde{x}}\right)$ (resp., $Z^{1}\left(G_{K}, I_{\tilde{x}}\right)$ ) the set of (continuous) 1-cocycles of $G_{K}^{\dagger}$ (resp., $G_{K}$ ) with coefficients in $I_{\tilde{x}}$, then it makes sense to refer to any element of $Z^{1}\left(G_{K}^{\dagger}, I_{\tilde{x}}\right)$ (resp., $Z^{1}\left(G_{K}, I_{\tilde{x}}\right)$ ) belonging to the inverse image of $a \in\left(K^{\times}\right)^{\wedge} \cong H^{1}\left(G_{K}^{\dagger}, I_{\tilde{x}}\right)$ (resp., $\cong H^{1}\left(G_{K}^{\dagger}, I_{\tilde{x}}\right)$ ), via the natural surjection, as a (continuous) 1-cocycle representing the Kummer class a.

\section{LEMMA 4.3}

For any choice $\left(C_{\tilde{x}}^{X}\right)$ (resp., $\left(C_{D}^{X}\right)$ ) of a specific decomposition group $D_{\tilde{x}} \subseteq \Pi_{\bar{X}_{x}^{\log }}$ (resp., $D_{X} \subseteq \Pi_{X_{2}}$ ), $I_{\tilde{x}}$ (resp., $I_{X}$ ) is normally terminal in $\Delta_{X_{2 / 1}}^{1}$ (see the Introduction), and $D_{\tilde{x}}^{(-)}$(resp., $\left.D_{X}^{(-)}\right)$-where the symbol $(-)$denotes either the presence or absence of $\dagger$-coincides with $N_{\Pi_{\bar{X}_{x}^{\log }}}\left(I_{\tilde{x}}\right)^{(-)}\left(\right.$resp., $\left.N_{\Pi_{X_{2}}}\left(I_{X}\right)^{(-)}\right)$(see the Introduction).

\section{Proof}

Recall that, by definition, we have $I_{\tilde{x}}=D_{\tilde{x}} \cap \Delta_{X_{2 / 1}}^{1} \subseteq \Pi_{X_{2}}$ and $I_{X}=D_{X} \cap$ $\Delta_{X_{2 / 1}}^{1} \subseteq \Pi_{X_{2}}$. Next, let us recall the well-known fact (see, e.g., [17, (2.3.1)]) that $I_{\tilde{x}}$ and $I_{X}$ are normally terminal (see the Introduction) in $\Delta_{X_{2 / 1}}^{1}$. Thus, the respective assertion follows immediately from the fact that $p_{1}^{\Pi}$ maps $D_{X}$ onto $\Pi_{X}$. On the other hand, the nonrespective assertion follows immediately from the observation that the images of $D_{\tilde{x}}$ and $\Pi_{\bar{X}_{x}^{\log }}$ coincide via $p_{1}^{\Pi} \circ i_{1}^{\Pi}$. This observation is a consequence of the geometry of the corresponding morphisms of $\log$ schemes, which implies that both of these images coincide with a decomposition group that is a subset of or equivalent to $\Pi_{X}$ associated to the point $x$.

\section{LEMMA 4.4}

(a) If we fix a choice $\left(C_{d}^{X}\right)$ of $d^{\Pi}: \Pi_{\mathbb{D}^{\log }} \rightarrow \Pi_{X_{2}}$, then there exists a unique choice $\left(C_{D}^{X}\right)$ of $D_{X} \subseteq \Pi_{X_{2}}$ such that the image of $d^{\Pi}$ coincides with $D_{X}$. By contrast, if we fix a choice $\left(C_{D}^{X}\right)$ of $D_{X} \subseteq \Pi_{X_{2}}$, then there exists a (not necessarily unique) choice $\left(C_{d}^{X}\right)$ of $d^{\Pi}: \Pi_{\mathbb{D}^{\log }} \rightarrow \Pi_{X_{2}}$ such that the image of $d^{\Pi}$ coincides with $D_{X}$.

(b) If we fix a triple of choices $\left(C_{i_{1}}^{X}\right),\left(C_{p_{1}}^{X}\right)$, and $\left(C_{\tilde{x}}^{X}\right)$, then there exists a unique pair consisting of a choice $\left(C_{p_{2}}^{X}\right)$ of $p_{2}^{\Pi}: \Pi_{X_{2}} \rightarrow \Pi_{X}$ and a choice $\left(C_{D}^{X}\right)$ of $D_{X} \subseteq \Pi_{X_{2}}$ that satisfy the following conditions.

(1) The image of the inertia group $I_{X} \subseteq D_{X}$ in $\Pi_{X_{2}}$ coincides with the image of $I_{\tilde{x}}$ via $i_{1}^{\Pi}$.

(2) The homomorphism $\left(p_{1}^{\Pi}, p_{2}^{\Pi}\right): \Pi_{X_{2}} \rightarrow \Pi_{X} \times_{G_{K}} \Pi_{X}$ maps $D_{X}$ onto the image of the diagonal embedding $\Pi_{X} \hookrightarrow \Pi_{X} \times_{G_{K}} \Pi_{X}$. 
Proof

Assertion (a) follows immediately from the definitions of $\Pi_{\mathbb{D}^{\log }}$ and $D_{X}$. Next, we consider assertion (b). First, let us observe that it follows immediately from the various definitions involved that $I_{X}$ and $I_{\tilde{x}}$ are $\Pi_{X_{2}}$-conjugate. Since, by Lemma $4.3, D_{X}$ coincides with the normalizer of $I_{X}$ in $\Pi_{X_{2}}$, it suffices to take $D_{X}$ to be the normalizer of $I_{\tilde{x}}$ in $\Pi_{X_{2}}$ and $p_{2}^{\Pi}$ to be such that the condition $p_{1}^{\Pi}=p_{2}^{\Pi}$ is satisfied on $D_{X}$. Uniqueness follows immediately from conditions (1) and (2) and the surjectivity of the restriction of $p_{2}^{\Pi}$ to $D_{X}$.

\section{PROPOSITION 4.5}

If we fix arbitrary choices $\left(C_{i_{1}}^{X}\right),\left(C_{p_{1}}^{X}\right),\left(C_{\tilde{x}}^{X}\right),\left(C_{\sigma}^{\dagger X}\right)$, and $\left(C_{\delta}^{X}\right)$, then there exists a unique triple of choices consisting of $\left(C_{p_{2}}^{X}\right),\left(C_{D}^{X}\right)$, and a choice of a specific automorphism induced by $s: \bar{X}_{2}^{\log } \stackrel{\sim}{\rightarrow} \bar{X}_{2}^{\log }$

$$
s^{\dagger}: \Pi_{X_{2}}^{\dagger} \stackrel{\sim}{\longrightarrow} \Pi_{X_{2}}^{\dagger}
$$

- which we shall denote by $\left(C_{s}^{\dagger X}\right)$-satisfying the two conditions (1) and (2) stated in Lemma 4.4(b), as well as the following conditions.

(1) The morphism $\bar{s}^{\dagger}: \Pi_{X}^{\dagger} \times_{G_{K}^{\dagger}} \Pi_{X}^{\dagger} \stackrel{\sim}{\longrightarrow} \Pi_{X}^{\dagger} \times_{G_{K}^{\dagger}} \Pi_{X}^{\dagger}$ induced by passing to the quotient $\Pi_{X_{2}}^{\dagger} \stackrel{p^{\dagger}}{\rightarrow} \Pi_{X}^{\dagger} \times_{G_{K}^{\dagger}} \Pi_{X}^{\dagger}$ determined by $p_{1}^{\Pi}, p_{2}^{\Pi}$ coincides with the morphism obtained by switching the two factors.

(2) $s^{\dagger}$ preserves $D_{X}^{\dagger} \subseteq \Pi_{X_{2}}^{\dagger}$, and the restriction $\left.s^{\dagger}\right|_{D_{X}^{\dagger}}: D_{X}^{\dagger} \stackrel{\sim}{\rightarrow} D_{X}^{\dagger}$ corresponds to an automorphism induced by $s_{\mathbb{D}}: \mathbb{D}^{\log } \stackrel{\sim}{\rightarrow} \mathbb{D}^{\log }$ via the identification $\Pi_{\mathbb{D}^{\log }}^{\dagger} \stackrel{\sim}{\rightarrow} D_{X}^{\dagger}$ determined by some choice of a specific homomorphism $d^{\Pi}: \Pi_{\mathbb{D}^{\log }} \rightarrow$ $\Pi_{X_{2}}$ whose image coincides with $D_{X}$ (see Lemma 4.4(a)).

(3) The continuous function $G_{K}^{\dagger} \rightarrow \Pi_{X_{2}}^{\dagger}$ defined by

$$
g \mapsto\left(s^{\dagger} \circ \sigma^{\dagger}\right)(g) \cdot \sigma^{\dagger}(g)^{-1}
$$

is valued in $I_{\tilde{x}} \subseteq \Pi_{X_{2}}^{\dagger}$ and coincides with the $\delta$ determined by $\left(C_{\delta}^{X}\right)$.

In particular, $s^{\dagger}$ induces the identity morphism on $I_{X} \subseteq \Pi_{X_{2}}^{\dagger}$.

Proof

We begin by proving the existence portion. Let us consider the following (not necessarily commutative) diagram

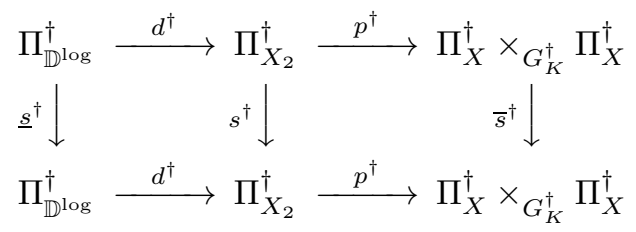

induced by $\left((*)^{\Pi}\right)$ and consisting of the horizontal arrows arising from the choice $\left(C_{p_{1}}^{X}\right)$ fixed in advance, the pair of choices $\left(C_{p_{2}}^{X}\right),\left(C_{d}^{X}\right)$ obtained by applying Lemmas 4.4(a) and 4.4(b), and arbitrary choices of the vertical arrows. By the surjectivity of $p^{\dagger}$, we can take $s^{\dagger}, \bar{s}^{\dagger}$ such that the right-hand square of the 
diagram $\left((*)^{\dagger}\right)$ commutes, and condition (1) is satisfied. The commutativity of the rectangle in $\left((*)^{\dagger}\right)$ up to conjugacy implies that there exists $\lambda \in \Pi_{X}^{\dagger} \times_{G_{K}^{\dagger}} \Pi_{X}^{\dagger}$ such that $\bar{s}^{\dagger} \circ\left(p^{\dagger} \circ d^{\dagger}\right)=\operatorname{Inn}(\lambda) \circ\left(p^{\dagger} \circ d^{\dagger}\right) \circ \underline{s}^{\dagger}$ (where $\operatorname{Inn}(\lambda)$ denotes the inner automorphism obtained by conjugating by $\lambda$ ). By the construction of the choice $\left(C_{D}^{X}\right)$ (see Lemma 4.4(b.2)), $p^{\dagger} \circ d^{\dagger}$ maps $\Pi_{\mathbb{D}^{\log }}^{\dagger}$ onto the subgroup of diagonal elements of $\Pi_{X}^{\dagger} \times{ }_{G_{K}^{\dagger}} \Pi_{X}^{\dagger}$; thus, $\operatorname{Inn}(\lambda)$ preserves this diagonal subgroup. Since $\Pi_{X}^{\dagger}$ is center-free (by Proposition 3.4(c)), we thus conclude that $\lambda$ is a diagonal element. Thus, by taking a lifting $\tilde{\lambda} \in \Pi_{\mathbb{D} \log }^{\dagger}$ of $\lambda$ and replacing $\underline{s}^{\dagger}$ by $\operatorname{Inn}\left(\tilde{\lambda}^{-1}\right) \circ$ $\underline{s}^{\dagger}$, we can make the rectangle in $\left((*)^{\dagger}\right)$ commute in the strict sense. Next, we observe (by applying again the commutativity of the rectangle in $\left((*)^{\dagger}\right)$ up to conjugacy) that $s^{\dagger} \circ d^{\dagger}=\operatorname{Inn}(\mu) \circ d^{\dagger} \circ \underline{s}^{\dagger}$ for some $\mu \in \Pi_{X_{2}}^{\dagger}$. By the commutativity of the rectangle in $\left((*)^{\dagger}\right), \mu$ projects via $p^{\dagger}$ into the center of $\Pi_{X}^{\dagger} \times_{G_{K}^{\dagger}} \Pi_{X}^{\dagger}$ and, hence (by Proposition 3.4(c)), to the unit element. Therefore, by replacing $s^{\dagger}$ by $\operatorname{Inn}\left(\mu^{-1}\right) \circ s^{\dagger}$, we conclude that we may choose $\underline{s}^{\dagger}, s^{\dagger}$, and $\bar{s}^{\dagger}$ so that the diagram $\left((*)^{\dagger}\right)$ commutes, and moreover, conditions (1) and (2) are satisfied.

Next, observe that by restricting $s^{\dagger}$ to $D_{X}^{\dagger}$, we obtain a commutative diagram

$$
\begin{aligned}
1 \longrightarrow I_{X} \longrightarrow D_{X}^{\dagger} \stackrel{\left.p^{\dagger}\right|_{D_{X}} ^{\dagger}}{\longrightarrow} \Pi_{X}^{\dagger} \longrightarrow 1 \\
\left.\left.\left.s^{\dagger}\right|_{I_{X}}\right|^{2} \longrightarrow s^{\dagger}\right|_{D_{X}^{\dagger} \downarrow^{2}} \longrightarrow \text { id }\left.\right|^{2} \\
1 \longrightarrow D_{X} \longrightarrow 1
\end{aligned}
$$

in which the right-hand vertical arrow is the identity automorphism of $\Pi_{X}^{\dagger}$. Write $\mathbb{M} \subseteq \mathbb{Q}$ for the monoid of positive rational numbers with l-power denominators, and write $\mathcal{N}$ for the monoid of global sections of the sheaf of monoids defining the $\log$ structure on a universal geometrically pro- $l$ két covering (see [6, Definition 3.1]) of $x^{\log } \times \bar{X}^{\log } \mathbb{D}^{\log }$. When $x \in X$ (resp., $\left.x \in \bar{X} \backslash X\right), \mathcal{N}$ admits a direct sum decomposition $\mathcal{N} \cong \mathbb{M} \oplus \bar{K}^{\times}$(resp., $\mathcal{N} \cong \mathbb{M} \oplus \mathbb{M} \oplus \bar{K}^{\times}$), where (see Remark 4.2.1(a)) the first factor (resp., first two factors) of the direct sum arise(s) from extracting roots of a local uniformizer of the divisor $\mathbb{D} \subseteq \bar{X}_{2}$ at $\tilde{x}$ (resp., of local uniformizers of the two irreducible divisors defining the log structure of $\bar{X}_{2}^{\log }$ at $\tilde{x})$ in a fashion compatible with the choice $\left(C_{\sigma}^{\dagger X}\right)$ of $\sigma$. Here, in the respective case, we assume that the first factor $\mathbb{M}$ corresponds to the divisor $\mathbb{D} \subseteq \bar{X}_{2}$. Next, observe that it follows from Lemma 4.1(b), together with the well-known local structure of $\bar{X}_{2}$ in a neighborhood of $\tilde{x}$, that the automorphism of $\mathcal{N} \cong \mathbb{M} \oplus \bar{K}^{\times}$ (resp., $\mathcal{N} \cong \mathbb{M} \oplus \mathbb{M} \oplus \bar{K}^{\times}$) induced by the automorphism $\underline{s}^{\dagger}$ of $\Pi_{\mathbb{D}^{\log }}^{\dagger}$ may be expressed in the form

$$
\begin{aligned}
\left(\frac{a}{l^{m}}, k\right) & \mapsto\left(\frac{a}{l^{m}},(-1)^{a / l^{m}} \cdot k\right) \\
\left(\text { resp., }\left(\frac{a_{1}}{l^{m_{1}}}, \frac{a_{2}}{l^{m_{2}}}, k\right)\right. & \left.\mapsto\left(\frac{a_{1}}{l^{m_{1}}}, \frac{a_{2}}{l^{m_{2}}},(-1)^{a_{1} / l^{m_{1}}} \cdot k\right)\right)
\end{aligned}
$$


for a suitable choice of a projective system $\left\{(-1)^{1 / l^{m}}\right\}_{m \in \mathbb{Z}_{\geq 0}}$ of $l$-power roots of -1 . In particular, we conclude that the restriction $\left.s^{\dagger}\right|_{I_{X}}$ is the identity morphism, and that the 1-cocycle $G_{K}^{\dagger} \ni g \mapsto\left(s^{\dagger} \circ \sigma^{\dagger}\right)(g) \cdot \sigma^{\dagger}(g)^{-1}$ is valued in $I_{X}=I_{\tilde{x}}$ (see Lemma 4.4(b.1)). Therefore, by replacing $\underline{s}^{\dagger}, s^{\dagger}$ by their composites with a suitable $I_{X}$-inner automorphism, we may assume that condition (3) is satisfied. This completes the proof of the existence assertion.

Next we prove the uniqueness portion. If $s_{1}^{\dagger}, s_{2}^{\dagger}$ are two maps that satisfy conditions (1), (2), and (3), then $s_{1}^{\dagger} \circ\left(s_{2}^{\dagger}\right)^{-1}=\operatorname{Inn}(\eta) \in \operatorname{Aut}\left(\Pi_{X_{2}}^{\dagger}\right)$ for some $\eta \in \Pi_{X_{2}}^{\dagger}$, and it follows from condition (2) that $\operatorname{Inn}(\eta)$ preserves the subgroup $D_{X}^{\dagger} \subseteq \Pi_{X_{2}}^{\dagger}$. Since $D_{X}^{\dagger}$ is normally terminal in $\Pi_{X_{2}}^{\dagger}$ (see Lemma 4.3), we thus conclude that $\eta$ is in $D_{X}^{\dagger}$. Moreover, it follows from condition (1) and the fact that $\Pi_{X}^{\dagger}$ is centerfree (see Proposition 3.4(c)) that $\eta$ lies in $\operatorname{Ker}\left(D_{X}^{\dagger} \stackrel{\left.p^{\dagger}\right|_{D_{X}^{\dagger}} ^{\dagger}}{\rightarrow} \Pi_{X}^{\dagger} \times_{G_{K}^{\dagger}} \Pi_{X}^{\dagger}\right.$ ), that is, $\eta \in I_{X}$. On the other hand, since the section $\sigma^{\dagger}$ acts faithfully on $I_{X}$ via the cyclotomic character, condition (3) implies that $\eta$ is the unit element, that is, that $s_{1}^{\dagger}=s_{2}^{\dagger}$.

\section{REMARK 4.5.1}

In the case $l \neq 2,-1$ coincides with the unit element 1 in $\left(K^{\times}\right)^{\wedge}$. Therefore, in the statement of Proposition 4.5 , by taking the choice $\left(C_{\delta}^{\dagger}\right)$ to be such that the 1-cocycle map $\delta$ is trivial, we may obtain an $s^{\dagger}$ satisfying $s^{\dagger} \circ \sigma^{\dagger}=\sigma^{\dagger}$.

\section{The proof of Theorem $A$}

This section is devoted to proving Theorem A. We begin with a review of the notation and setup. Let $l$ be a prime number, let $K$ be a finite field in which $l$ is invertible, and let $\bar{K}$ be a separable closure of $K$. We shall denote by $G_{K}$ the Galois group of $\bar{K}$ over $K$. Next, let $X$ be a hyperbolic curve over $K$ of type $\left(g_{X}, r_{X}\right)$, and let $x^{\log }$ be a strict $K$-rational $\log$ point of $\bar{X}^{\log }:=\bar{X}_{1}^{\log }$; write $\bar{X}_{x}^{\log }:=x^{\log } \times \bar{X}_{\bar{X}^{\log }, p_{1}^{\log }} \bar{X}_{2}^{\log }, \tilde{x}^{\log }:=x^{\log } \times_{\bar{X}^{\log }} \mathbb{D}^{\log }$. In addition, we assume that we have fixed choices $\left(C_{i_{1}}^{X}\right),\left(C_{p_{1}}^{X}\right),\left(C_{\tilde{x}}^{X}\right),\left(C_{\sigma}^{X}\right),\left(C_{\delta}^{X}\right)$ (i.e., in the sense that they are not subject to conjugacy indeterminacy).

As a first step, we define two actions of $G_{K}$ on various topological groups, graded Lie algebras, and linear algebraic groups associated to the fundamental groups of $\bar{X}_{x}^{\log }$ and $X_{2}$. As we shall discuss in the following, these two actions are mapped to one another via the morphisms induced by the switching morphism obtained in Section 4.

\section{DEFINITION 5.1}

(a) The choice $\left(C_{\sigma}\right)$ of a section $\sigma: G_{K} \rightarrow D_{\tilde{x}}$ determines, by composing with the natural morphism $D_{\tilde{x}} \rightarrow \Pi_{\bar{X}_{x}^{\log }}$ (resp., $D_{\tilde{x}} \rightarrow \Pi_{X_{2}}, D_{\tilde{x}} \rightarrow \Pi_{X \times 2}$ ), a nat-

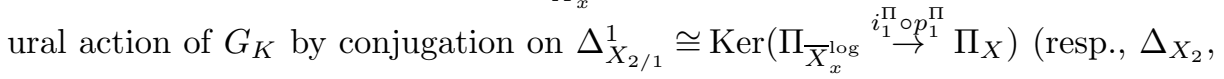


$\left.\Delta_{X \times 2}\right)$ and hence also on

$$
\begin{gathered}
\operatorname{Gr}_{X_{2 / 1}}^{1}:=\operatorname{Gr}_{\mathbb{Q}_{l}}\left(\Delta_{X_{2 / 1}}^{1}\right) \\
\left(\text { resp., } \operatorname{Gr}_{X_{2}}:=\operatorname{Gr}_{\mathbb{Q}_{l}}\left(\Delta_{X_{2}}\right), \operatorname{Gr}_{X^{\times 2}}:=\operatorname{Gr}_{\mathbb{Q}_{l}}\left(\Delta_{X \times 2}\right)\right), \\
\operatorname{Lie}_{X_{2 / 1}}^{1}:=\operatorname{Lie}\left(\Delta_{X_{2 / 1}}^{1}(1 / \infty)\right) \\
\left(\text { resp., } \operatorname{Lie}_{X_{2}}:=\operatorname{Lie}\left(\Delta_{X_{2}}(1 / \infty)\right), \operatorname{Lie}_{X^{\times 2}}:=\operatorname{Lie}\left(\Delta_{X^{\times 2}}(1 / \infty)\right)\right), \\
\operatorname{Lin}_{X_{2 / 1}}^{1}:=\operatorname{Lin}\left(\Delta_{X_{2 / 1}}^{1}(1 / \infty)\right)\left(\mathbb{Q}_{l}\right) \\
\left(\text { resp., } \operatorname{Lin}_{X_{2}}:=\operatorname{Lin}\left(\Delta_{X_{2}}(1 / \infty)\right)\left(\mathbb{Q}_{l}\right), \operatorname{Lin}_{X^{\times 2}}:=\operatorname{Lin}\left(\Delta_{X^{\times 2}}(1 / \infty)\right)\left(\mathbb{Q}_{l}\right)\right) .
\end{gathered}
$$

In the following, we regard these objects as being equipped with these $G_{K^{-}}$ actions. From the discussion in Definition 3.5(b), we have the following commutative diagram consisting of $G_{K}$-equivariant morphisms:

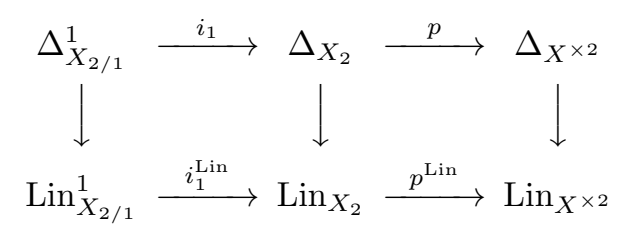

and topological groups equipped with $G_{K}$-actions

$$
\Delta_{X_{2}}^{\mathrm{Lie}}:=\Delta_{X^{\times 2}} \times_{\operatorname{Lin}_{X \times 2}} \operatorname{Lin}_{X_{2}}, \quad \Pi_{X_{2}}^{\mathrm{Lie}}:=\Delta_{X_{2}}^{\mathrm{Lie}} \rtimes G_{K}
$$

as well as $G_{K}$-equivariant homomorphisms of topological groups

$$
\operatorname{Int}_{X}^{\Delta}: \Delta_{X_{2}} \rightarrow \Delta_{X_{2}}^{\mathrm{Lie}}, \quad \operatorname{Int}_{X}^{\Pi}: \Pi_{X_{2}} \rightarrow \Pi_{X_{2}}^{\mathrm{Lie}}
$$

(b) Next, the choice $\left(C_{\sigma}\right),\left(C_{\delta}\right)$ yields a new section of the surjective homomorphism $D_{\tilde{x}} \rightarrow G_{K}$

$$
\begin{aligned}
\sigma_{\delta}: G_{K} & \longrightarrow D_{\tilde{x}}, \\
g & \mapsto \delta(g) \cdot \sigma(g),
\end{aligned}
$$

which is a homomorphism of topological groups. Then the section $\sigma_{\delta}$ determines, in a similar way to (a), a natural action of $G_{K}$ by conjugation on

$$
\begin{aligned}
& \breve{G r}_{X_{2 / 1}}^{1}:=\operatorname{Gr}_{\mathbb{Q}_{l}}\left(\Delta_{X_{2 / 1}}^{1}\right) \\
& \text { (resp., } \breve{G r}_{X_{2}}:=\operatorname{Gr}_{\mathbb{Q}_{l}}\left(\Delta_{X_{2}}\right), \breve{G r}_{X^{\times 2}}:=\operatorname{Gr}_{\mathbb{Q}_{l}}\left(\Delta_{X \times 2}\right) \text { ), } \\
& \breve{L i e}_{X_{2 / 1}}^{1}:=\operatorname{Lie}\left(\Delta_{X_{2 / 1}}^{1}(1 / \infty)\right) \\
& \left(\text { resp., } \breve{L i e}_{X_{2}}:=\operatorname{Lie}\left(\Delta_{X_{2}}(1 / \infty)\right), \breve{L i e}_{X \times 2}:=\operatorname{Lie}\left(\Delta_{X \times 2}(1 / \infty)\right)\right) \text {, } \\
& \breve{L i n}_{X_{2 / 1}}^{1}:=\operatorname{Lin}\left(\Delta_{X_{2 / 1}}^{1}(1 / \infty)\right)\left(\mathbb{Q}_{l}\right) \\
& \left(\text { resp., } \breve{L i n}_{X_{2}}:=\operatorname{Lin}\left(\Delta_{X_{2}}(1 / \infty)\right)\left(\mathbb{Q}_{l}\right), \breve{L i n}_{X^{\times 2}}:=\operatorname{Lin}\left(\Delta_{X^{\times 2}}(1 / \infty)\right)\left(\mathbb{Q}_{l}\right)\right) \text {, }
\end{aligned}
$$


where, in the following, we regard these objects as being equipped with the $G_{K^{-}}$ actions just defined, as well as topological groups equipped with $G_{K}$-actions

$$
\breve{\Delta} \breve{X}_{2}^{\text {Lie }}:=\Delta_{X \times 2} \times \breve{L i n}_{X \times 2} \breve{L i n}_{X_{2}}, \quad \breve{\Pi}_{X_{2}}^{\text {Lie }}:=\breve{\Delta}_{X_{2}}^{\text {Lie }} \rtimes G_{K} .
$$

Next, let us recall that by applying Proposition 4.5, together with the choices $\left(C_{i_{1}}\right),\left(C_{p_{1}}\right),\left(C_{\tilde{x}}\right),\left(C_{\delta}\right)$ and the choice $\left(C_{\sigma}^{\dagger}\right)$ determined naturally by $\left(C_{\sigma}\right)$, we obtain a choice $\left(C_{s}^{\dagger}\right)$ of a specific automorphism $s^{\dagger}: \Pi_{X_{2}}^{\dagger} \rightarrow \Pi_{X_{2}}^{\dagger}$. Let $s^{\Pi}: \Pi_{X_{2}} \stackrel{\sim}{\rightarrow}$ $\Pi_{X_{2}}$ be an automorphism that induces the outer automorphism determined by the switching morphism $s_{X}: \bar{X}_{2}^{\log } \rightarrow \bar{X}_{2}^{\log }$ and is compatible with $s^{\dagger}: \Pi_{X_{2}}^{\dagger} \rightarrow$ $\Pi_{X_{2}}^{\dagger}$. Then, by Lemma 5.2 below, we obtain $G_{K^{-}}$equivariant isomorphisms of topological groups

$$
s^{\Delta^{\text {Lie }}}: \Delta_{X_{2}}^{\text {Lie }} \stackrel{\sim}{\longrightarrow} \breve{\Delta}_{X_{2}}^{\text {Lie }}, \quad s^{\Pi^{\text {Lie }}}: \Pi_{X_{2}}^{\text {Lie }} \stackrel{\sim}{\longrightarrow} \breve{\Pi}_{X_{2}}^{\text {Lie }}
$$

induced by $s^{\Pi}$ and a (non- $G_{K}$-equivariant) commutative diagram as follows:

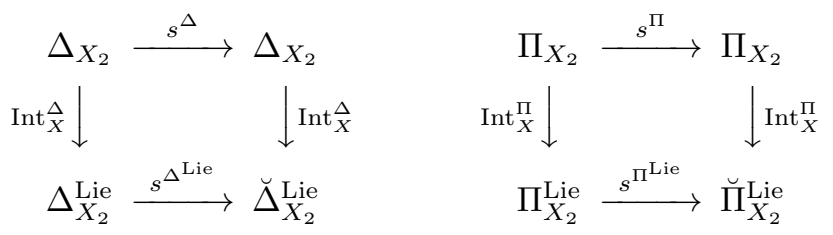

LEMMA 5.2

The $G_{K}$-action induced by $\sigma_{\delta}$ (see Definition 5.1(b)) on $\Delta_{X_{2}}$ (hence also on $\breve{G r}_{X_{2}}, \breve{L i e}_{X_{2}}, \breve{L i n}_{X_{2}}$, and $\breve{\Delta}_{X_{2}}^{\mathrm{Lie}}$ ) coincides with the action

$$
\begin{aligned}
G_{K} & \longrightarrow \operatorname{Aut}\left(\Delta_{X_{2}}\right), \\
g & \mapsto \operatorname{Inn}\left(s^{\Pi} \circ i_{1}^{\Pi} \circ \sigma(g)\right) .
\end{aligned}
$$

Proof

This follows immediately from Proposition 4.5(3), together with the definition of the $G_{K}$-action induced by $\sigma_{\delta}$.

\section{LEMMA 5.3}

We have that $\operatorname{Int}_{X}^{\Delta}$ and $\operatorname{Int}_{X}^{\Pi}$ are injective (see [2, Lemma 4.3] in the case where $X$ is proper).

\section{Proof}

It suffices to verify that $\Delta_{X_{2}} \rightarrow \operatorname{Lin}_{X_{2}}$ is injective. But this follows from the discussion in Definition 3.5(b) and the fact that $\bigcap_{m \geq 1} \Delta_{X \times 2}(m)=1$ (see [18, Corollary 2.6]).

Next, we shall construct certain graded Lie algebras equipped with a $G_{K}$-actionwhich we shall denote by $\mathcal{L}_{X}^{1}$ and $\mathcal{L}_{X}^{2}$-by using various subgroups of $\Pi_{\bar{X}_{x}^{\log }}$. Comparing these graded Lie algebras to the graded Lie algebras discussed above (see Lemmas 5.5, 5.6) will allow us to reconstruct various groups associated to 
$\Pi_{X_{2}}$ from those associated to $\Pi_{\bar{X}_{x}^{\log }}$ (see Proposition 5.8). This will play an important role in the proof of Theorem A.

\section{DEFINITION 5.4}

(a) For each $j=1,2, \ldots, r$, let us fix a choice of the inertia subgroup $I_{j} \subseteq$ $\Delta_{X_{2 / 1}}^{1} \cong \operatorname{Ker}\left(\Pi_{\bar{X}_{x}^{\log }} \stackrel{p_{1}^{\Pi} \circ i_{1}^{\Pi}}{\rightarrow} \Pi_{X}\right)$ associated to the $j$ th cusp (relative to some ordering of the cusps of $\left.X \times_{K} \bar{K}\right)$ among the various $\Delta_{X_{2 / 1}}^{1}$-conjugates of these subgroups. Then, we have canonical isomorphisms

$$
\eta_{j}: I_{\tilde{x}} \stackrel{\sim}{\longrightarrow} I_{j} \quad(j=1,2, \ldots, r) .
$$

Indeed, recall that the kernel of the natural quotient $\left(\Delta_{X_{2 / 1}}^{1}\right)^{\mathrm{ab}} \rightarrow \Delta_{\bar{X}}^{\mathrm{ab}}$ coincides with the submodule $\bigoplus_{j^{\prime}=1}^{r} I_{j^{\prime}} \subseteq\left(\Delta_{X_{2 / 1}}^{1}\right)^{\text {ab }}$; thus, since the subgroup $I_{\tilde{x}}$ of $\left(\Delta_{X_{2 / 1}}^{1}\right)^{\mathrm{ab}}$ is contained in this kernel, it follows that the composite $I_{\tilde{x}} \hookrightarrow$ $\bigoplus_{j^{\prime}=1}^{r} I_{j^{\prime}} \rightarrow I_{j} \stackrel{(-1)}{\rightarrow} I_{j}$ of this inclusion with the natural projection to the $j$ th factor multiplied by -1 yields the required isomorphism.

For $n=1,2$, we shall denote by $\mathcal{V}^{n}$ the completion with respect to the filtration topology of the free Lie algebra generated by

$$
V^{n}:=I_{\tilde{x}} \oplus\left(\bigoplus_{j=1}^{r} I_{j} \oplus \Delta_{\bar{X}}^{\mathrm{ab}}\right)^{\oplus n}
$$

equipped with a natural grading (hence also a filtration) by taking $I_{\tilde{x}}, I_{j}$ to be of weight 2 and $\Delta_{\bar{X}}^{\mathrm{ab}}$ to be of weight 1 .

(b) If $X$ has genus at least 1 , then we shall write

$$
M_{X}:=\operatorname{Hom}_{\mathbb{Z}_{l}}\left(H^{2}\left(\Delta_{\bar{X}}, \mathbb{Z}_{l}\right), \mathbb{Z}_{l}\right) .
$$

Note that $M_{X}$ is canonically isomorphic to $I_{\tilde{x}}$ as a $G_{K}$-module. Indeed, recall the natural quotient $\left(\Delta_{X_{2 / 1}}^{1} /\left\langle I_{j}\right\rangle_{j=1, \ldots, r}\right) \rightarrow \Delta_{\bar{X}}$; the associated maximal cuspidally central quotient (see [12, Definition 1.1(i)]) yields an extension of $\Delta_{\bar{X}}$ by $I_{\tilde{x}}$. This extension determines a generator of the rank one free $\mathbb{Z}_{l}$-module $H^{2}\left(\Delta_{\bar{X}}, I_{\tilde{x}}\right) \cong$ $\operatorname{Hom}_{\mathbb{Z}_{l}}\left(M_{X}, I_{\tilde{x}}\right)$ (see, e.g., [11, Lemmas 4.2(i), 4.2(ii), 4.2(iii)]) and, hence, an isomorphism $M_{X} \stackrel{\sim}{\rightarrow} I_{\tilde{x}}$, as desired.

The cup product on the group cohomology of $\Delta_{\bar{X}}$

$$
\bigwedge^{2} H^{1}\left(\Delta_{\bar{X}}, M_{X}\right) \longrightarrow H^{2}\left(\Delta_{\bar{X}}, M_{X} \otimes_{\mathbb{Z}_{l}} M_{X}\right) \cong M_{X}
$$

determines an isomorphism

$$
\left(H^{1}\left(\Delta_{\bar{X}}, M_{X}\right) \cong\right) \operatorname{Hom}\left(\Delta_{\bar{X}}^{\mathrm{ab}}, M_{X}\right) \stackrel{\sim}{\longrightarrow} \Delta_{\bar{X}}^{\mathrm{ab}}\left(\cong \operatorname{Hom}\left(H^{1}\left(\Delta_{\bar{X}}, M_{X}\right), M_{X}\right)\right)
$$

and, hence, composites of natural homomorphisms

$$
\phi: I_{\tilde{x}} \stackrel{\sim}{\longrightarrow} M_{X} \longrightarrow \bigwedge^{2} \Delta_{\bar{X}}^{\mathrm{ab}}, \quad \psi: \bigwedge^{2} \Delta_{\bar{X}}^{\mathrm{ab}} \longrightarrow M_{X} \stackrel{\sim}{\longrightarrow} I_{\tilde{x}} .
$$


If $X$ has genus 0 , then we take $\phi, \psi$ to be the zero maps.

(c) We define $\mathcal{L}_{X}^{n}$ to be the quotient of $\mathcal{V}^{n}$ by the relations determined by the images of the following morphisms (which are patterned after the presentations given in Proposition 3.9).

(1) When $n=1$,

- $I_{\tilde{x}} \longrightarrow \mathcal{V}^{1}(2 / 3) ; m \mapsto\left(\operatorname{id}_{I_{\tilde{x}}}+\sum \eta_{j}+\phi\right)(m)$.

(2) When $n=2\left(1 \leq i \leq g, 1 \leq j, j^{\prime} \leq r, j \neq j^{\prime},\left\{k, k^{\prime}\right\}=\{1,2\}\right)$,

- $I_{\tilde{x}} \longrightarrow \mathcal{V}^{2}(2 / 3) ; m \mapsto m+i_{k}\left(\sum \eta_{j}+\phi\right)(m)$;

- $I_{\tilde{x}} \otimes_{\mathbb{Z}_{l}} \Delta_{\bar{X}}^{\mathrm{ab}} \longrightarrow \mathcal{V}^{2}(3 / 4) ; m \otimes a \mapsto\left[i_{k} \circ \eta_{j}(m), i_{k^{\prime}}(a)\right] ;$

- $I_{\tilde{x}} \longrightarrow \mathcal{V}^{2}(4 / 5) ; m \mapsto\left[i_{k} \circ \eta_{j}(m), i_{k^{\prime}} \circ \eta_{j^{\prime}}(m)\right]$;

- $\bigwedge^{2} \Delta_{\bar{X}}^{\mathrm{ab}} \longrightarrow \mathcal{V}^{2}(2 / 3) ; a \wedge a^{\prime} \mapsto\left[i_{k}(a), i_{k^{\prime}}\left(a^{\prime}\right)\right]-\psi\left(a \wedge a^{\prime}\right)$,

where $[\cdot, \cdot]$ denotes the Lie bracket, and for $k=1,2, i_{k}:\left(\bigoplus I_{j} \oplus \bigwedge^{2} \Delta_{\bar{X}}^{\mathrm{ab}}\right) \hookrightarrow$ $\left(\bigoplus I_{j} \oplus \bigwedge^{2} \Delta_{\bar{X}}^{\mathrm{ab}}\right)^{\oplus 2}$ denotes the inclusion into the $k$ th factor.

(d) The natural $G_{K}$-action on each direct summand in $\mathcal{V}^{n}$ determines a natural $G_{K}$-action on $\mathcal{V}^{n}$. One verifies immediately that the ideal generated by the relations defined in (c) is preserved by this $G_{K}$-action. Thus, we obtain a natural $G_{K}$-action on the graded Lie algebra

$$
\mathcal{L}_{X}^{1} \quad\left(\text { resp. }, \mathcal{L}_{X}^{2}\right)
$$

and a $G_{K}$-equivariant homomorphism

$$
i_{1}^{\mathcal{L}}: \mathcal{L}_{X}^{1} \longrightarrow \mathcal{L}_{X}^{2}
$$

of graded Lie algebras determined by the map on generators given by

$$
\begin{aligned}
I_{\tilde{x}} \oplus\left(\bigoplus_{j=1}^{r} I_{j} \oplus \Delta_{\bar{X}}^{\mathrm{ab}}\right) & \longrightarrow I_{\tilde{x}} \oplus\left(\bigoplus_{j=1}^{r} I_{j} \oplus \Delta_{\tilde{X}}^{\mathrm{ab}}\right)^{\oplus 2}, \\
(a, b) & \mapsto\left(a, i_{1}(b)\right),
\end{aligned}
$$

as well as a $G_{K}$-equivariant isomorphism

$$
s_{X}^{\mathcal{L}}: \mathcal{L}_{X}^{2} \stackrel{\sim}{\longrightarrow} \mathcal{L}_{X}^{2}
$$

of graded Lie algebras determined by the map on generators given by

$$
\begin{aligned}
I_{\tilde{x}} \oplus\left(\bigoplus_{j=1}^{r} I_{j} \oplus \Delta_{\bar{X}}^{\mathrm{ab}}\right)^{\oplus 2} & \longrightarrow I_{\tilde{x}} \oplus\left(\bigoplus_{j=1}^{r} I_{j} \oplus \Delta_{\bar{X}}^{\mathrm{ab}}\right)^{\oplus 2}, \\
\left(a, b_{1}, b_{2}\right) & \mapsto\left(a, b_{2}, b_{1}\right) .
\end{aligned}
$$

\section{LEMMA 5.5}

Consider the homomorphism of graded Lie algebras $\mathcal{V}^{1} \rightarrow \operatorname{Gr}_{\mathbb{Q}_{l}}\left(\Delta_{X_{2 / 1}}^{1}\right)$ determined by the natural inclusions $\Delta_{\bar{X}}^{\mathrm{ab}} \hookrightarrow \operatorname{Gr}_{\mathbb{Q}_{l}}\left(\Delta_{X_{2 / 1}}^{1}\right)(1 / 2), I_{\tilde{x}} \hookrightarrow \operatorname{Gr}_{\mathbb{Q}_{l}}\left(\Delta_{X_{2 / 1}}^{1}\right)(2 /$ $3)$, and $I_{j} \hookrightarrow \operatorname{Gr}_{\mathbb{Q}_{l}}\left(\Delta_{X_{2 / 1}}^{1}\right)(2 / 3)$. This homomorphism of graded Lie algebras fac- 
tors through $\mathcal{L}_{X}^{1}$, and the resulting homomorphism $\mathfrak{h}^{1}: \mathcal{L}_{X}^{1} \rightarrow \operatorname{Gr}_{\mathbb{Q}_{l}}\left(\Delta_{X_{2 / 1}}^{1}\right)$ is a $G_{K}$-equivariant isomorphism of graded Lie algebras, whether we regard $\operatorname{Gr}_{\mathbb{Q}_{l}}\left(\Delta_{X_{2 / 1}}^{1}\right)$ as the underlying graded Lie algebra (i.e., without $G_{K}$-action) of $\mathrm{Gr}_{X_{2 / 1}}^{1}$ or as the underlying graded Lie algebra of $\breve{G r}_{X_{2 / 1}}^{1}$.

\section{Proof}

The asserted $G_{K}$-equivariance follows immediately from the definitions. Thus, it suffices to verify that $\mathfrak{h}^{1}$ is an isomorphism. When $x$ is not a cusp of $X$, this follows immediately from Proposition 3.9(a) applied to $X_{x}$. Thus, it suffices to verify that $\mathfrak{h}^{1}$ is an isomorphism in the case where $x$ is a cusp of $X$. Let $S$ be a mixed characteristic trait (i.e., the spectrum of a Henselian discrete valuation ring) whose residue field is isomorphic to the residue field of $x$, and write $S^{\text {log }}$ for the $\log$ scheme obtained by equipping $S$ with the $\log$ structure determined by the closed point of $S$. Next, let us take a stable $\log$ curve $\bar{X}_{S}^{\log } \rightarrow S^{\log }$ whose special fiber is isomorphic to $\bar{X}_{x}^{\log } \rightarrow x^{\log }$ and such that the interior $U$ of $X_{S}^{\log }$ is a hyperbolic curve over the fraction field of $S$. Then (see the discussion from $[14$, Section 0] in the characteristic zero case) we obtain a natural isomorphism $\Delta_{\bar{X}_{x}^{\log }} \stackrel{\sim}{\rightarrow} \Delta_{U}$ by composing a certain specialization isomorphism $\Delta_{\bar{X}_{x}^{\log }} \stackrel{\sim}{\rightarrow} \Delta_{\bar{X}_{S}^{\log }}$ with an isomorphism $\Delta_{\bar{X}_{S} \log } \stackrel{\sim}{\rightarrow} \Delta_{U}$ arising from the log purity theorem. Hence, the fact that $\mathfrak{h}^{1}$ is an isomorphism follows immediately by applying this isomorphism $\Delta_{\bar{X}_{x}^{\log }} \stackrel{\sim}{\rightarrow} \Delta_{U}$ together with Proposition 3.9(a).

\section{LEMMA 5.6}

Let

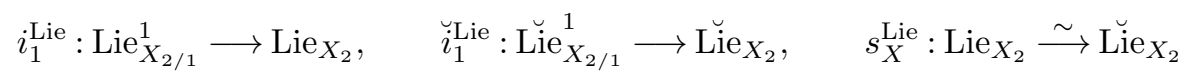

be the $G_{K}$-equivariant homomorphisms of graded Lie algebras induced by $i_{1}^{\Pi}$ : $\Pi_{\bar{X}_{x}^{\log }} \rightarrow \Pi_{X_{2}}, i_{1}^{\Pi}: \Pi_{\bar{X}_{x}^{\log }} \rightarrow \Pi_{X_{2}}$, and $s_{X}^{\Pi}: \Pi_{X_{2}} \stackrel{\sim}{\rightarrow} \Pi_{X_{2}}$, respectively. Then there exist $G_{K}$-equivariant isomorphisms of graded Lie algebras

$$
\begin{array}{ll}
h_{X}^{1}: \mathcal{L}_{X}^{1} \stackrel{\sim}{\longrightarrow} \operatorname{Lie}_{X_{2 / 1}}^{1}, & \breve{h}_{X}^{1}: \mathcal{L}_{X}^{1} \stackrel{\sim}{\longrightarrow} \breve{L i e}_{X_{2 / 1}}^{1}, \\
h_{X}^{2}: \mathcal{L}_{X}^{2} \stackrel{\sim}{\longrightarrow} \operatorname{Lie}_{X_{2}}, \quad \breve{h}_{X}^{2}: \mathcal{L}_{X}^{2} \stackrel{\sim}{\longrightarrow} \breve{L i e}_{X_{2}}
\end{array}
$$

which fit into the following commutative diagrams consisting of $G_{K}$-equivariant morphisms:

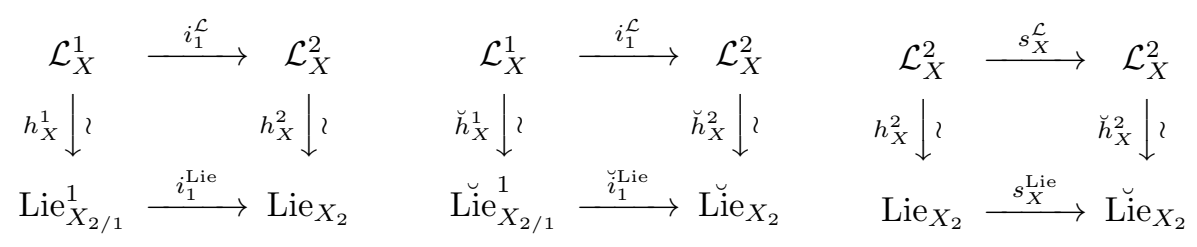


Proof

Consider the homomorphism of graded Lie algebras $\mathcal{V}^{2} \rightarrow \mathrm{Gr}_{\mathbb{Q}_{l}}\left(\Delta_{X_{2}}\right)$ determined by

$$
\begin{aligned}
I_{\tilde{x}} \oplus\left(\bigoplus_{j=1}^{r} I_{j} \oplus \Delta_{X}^{\mathrm{ab}}\right)^{\oplus 2} & \longrightarrow \operatorname{Gr}_{\mathbb{Q}_{l}}\left(\Delta_{X_{2}}\right), \\
\left(a, b_{1}, b_{2}\right) & \mapsto i_{1}^{\Pi}\left(a+b_{1}\right)+s_{X}^{\Pi} \circ i_{1}^{\Pi}\left(b_{2}\right) .
\end{aligned}
$$

Then it follows from Proposition 3.9(b) that this homomorphism of graded Lie algebras factors through $\mathcal{L}_{X}^{2}$, and that the resulting homomorphism $\mathfrak{h}^{2}: \mathcal{L}_{X}^{2} \rightarrow$ $\operatorname{Gr}_{\mathbb{Q}_{l}}\left(\Delta_{X_{2}}\right)$ is a $G_{K}$-equivariant isomorphism of graded Lie algebras, whether we regard $\mathfrak{h}^{2}$ as the morphism of underlying graded Lie algebras (i.e., without $G_{K^{-}}$ actions) $h^{2}: \mathcal{L}_{X}^{2} \rightarrow \mathrm{Gr}_{X_{2}}$ or as the morphism of underlying graded Lie algebras $\breve{h}^{2}: \mathcal{L}_{X}^{2} \rightarrow \breve{G r}_{X_{2}}$. If we denote by $i_{1}^{\mathrm{Gr}}: \mathrm{Gr}_{X_{2 / 1}}^{1} \rightarrow \mathrm{Gr}_{X_{2}}, \breve{i}_{1}^{\mathrm{Gr}}: \breve{G r}_{X_{2 / 1}}^{1} \rightarrow \breve{G r}_{X_{2}}$, and $s_{X}^{\mathrm{Gr}}: \mathrm{Gr}_{X_{2}} \stackrel{\sim}{\rightarrow} \breve{G r}_{X_{2}}$ the $G_{K}$-equivariant homomorphisms of graded Lie algebras induced by $i_{1}^{\Pi}: \Pi_{\bar{X}_{x}^{\log }}^{\rightarrow} \Pi_{X_{2}}, i_{1}^{\Pi}: \Pi_{\bar{X}_{x}^{\log }} \rightarrow \Pi_{X_{2}}$, and $s_{X}^{\Pi}: \Pi_{X_{2}} \stackrel{\sim}{\rightarrow} \Pi_{X_{2}}$, respectively, then we obtain $G_{K}$-equivariant commutative diagrams as follows:

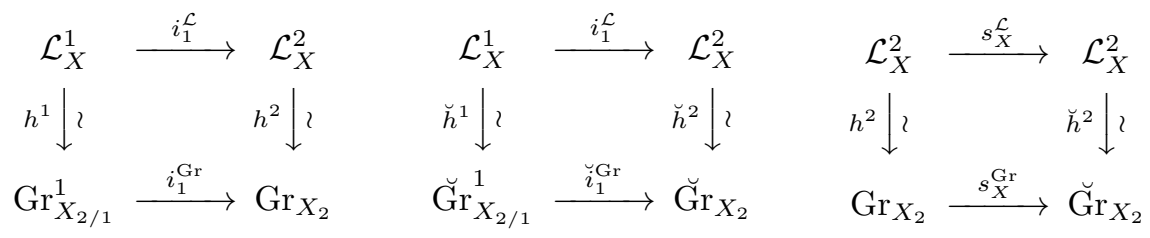

On the other hand, it follows from Proposition 3.8 that we have $G_{K}$-equivariant commutative diagrams as follows:

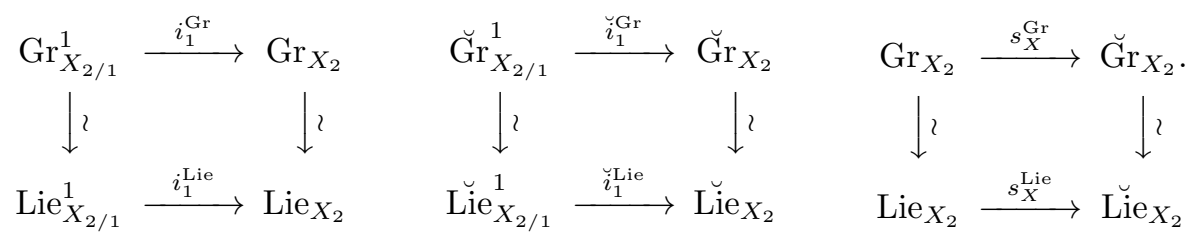

By composing the vertical arrows in these commutative diagrams, we obtain the required isomorphisms.

Now, let $L$ be a finite field of cardinality prime to $l$, let $Y$ be a hyperbolic curve over $L$ of type $\left(g_{Y}, r_{Y}\right)$, and let $y^{\log }$ be a strict $L$-rational log point of $\bar{Y}^{\log }:=\bar{Y}_{1}^{\log }$. We shall use notation for objects obtained from $Y$ (e.g., $Y_{2}, \bar{Y}_{y}^{\log }$, $\left.\Pi_{Y_{2}}, \Pi_{\bar{Y}_{y}^{\log }}\right)$ that is similar to that from $X$.

\section{DEFINITION 5.7}

(a) Consider an isomorphism of profinite groups $\alpha: \Pi_{X} \stackrel{\sim}{\rightarrow} \Pi_{Y}$ (resp., $\Pi_{\bar{X}_{x}^{\log }} \stackrel{\sim}{\rightarrow} \Pi_{\bar{Y}_{y}^{\log }}$ ). Then the natural surjections $\Pi_{X} \rightarrow G_{K}, \Pi_{Y} \rightarrow G_{L}$ (resp., $\left.\Pi_{\bar{X}_{x}^{\log }} \rightarrow G_{K}, \Pi_{\bar{Y}_{x}^{\log }} \rightarrow G_{L}\right)$ arising from the structure morphisms over finite fields may be characterized group-theoretically (see [20, Proposition 3.3]) as 
the (unique) maximal ( $\hat{\mathbb{Z}}_{\text {- }}$ free abelian quotient. Thus, $\alpha$ induces an isomorphism $G_{K} \stackrel{\sim}{\rightarrow} G_{L}$. We shall say that $\alpha$ is Frobenius-preserving if the isomorphism $G_{K} \stackrel{\sim}{\rightarrow} G_{L}$ obtained as above preserves the Frobenius elements.

(b) We shall denote by

$$
\left(C_{x, y}^{X \stackrel{\sim}{\rightarrow} Y}\right) \quad\left(\text { resp. },\left(C_{x, y}^{\bar{X}_{x}^{\log } \stackrel{\sim}{\rightarrow} \bar{Y}_{y}^{\log }}\right)\right)
$$

a choice of a specific Frobenius-preserving isomorphism $\Pi_{X} \stackrel{\sim}{\rightarrow} \Pi_{Y}$ (resp., $\Pi_{\bar{X}_{x}^{\log }} \stackrel{\sim}{\rightarrow}$ $\Pi_{\bar{Y}_{y}^{\log }}$ ) which maps the decomposition group of $x$ (resp., the diagonal cusp $\tilde{x}$ ) onto the decomposition group of $y$ (resp., the diagonal cusp $\tilde{y}$ ) up to conjugation.

\section{PROPOSITION 5.8}

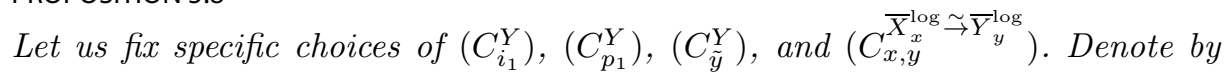
$\alpha: \Pi_{\bar{X}_{x}^{\log }} \stackrel{\sim}{\rightarrow} \Pi_{\bar{Y}_{y}^{\log }}$ the isomorphism determined by $\left(C_{x, y}^{\bar{X}_{x}^{\log } \stackrel{\sim}{\rightarrow} \bar{Y}_{y}^{\log }}\right)$. Let us assume that the decomposition subgroups determined by $\left(C_{\tilde{x}}^{X}\right),\left(C_{\tilde{y}}^{Y}\right)$ are compatible with respect to $\alpha$.

(a) There exists a unique pair consisting of a choice $\left(C_{\theta}^{Y}\right)$ of a specific 1-cocycle $\theta: G_{L} \rightarrow I_{\tilde{y}}:=\operatorname{Ker}\left(D_{\tilde{y}} \rightarrow \Pi_{Y}\right)$ and a choice $\left(C_{\tau}^{Y}\right)$ of a specific section $\tau: G_{L} \rightarrow D_{\tilde{y}}$ which are compatible with $\left(C_{\delta}^{X}\right)$ and $\left(C_{\sigma}^{X}\right)$, respectively, via $\left(C_{x, y}^{X \underset{\sim}{\sim} Y}\right)$ in an evident fashion.

(b) There exists a $G_{K}$-equivariant isomorphism $\alpha_{2}^{\Delta^{\text {Lie }}}: \Delta_{X_{2}}^{\text {Lie }} \stackrel{\sim}{\rightarrow} \Delta_{Y_{2}}^{\text {Lie }}$ of topological groups satisfying the following conditions.

(1) $\alpha_{2}^{\Delta^{\text {Lie }}}$ is also $G_{K}$-equivariant when we regard it as a map $\breve{\Delta}_{X_{2}}^{\text {Lie }} \stackrel{\sim}{\rightarrow} \breve{\Delta}_{Y_{2}}^{\text {Lie }}$ under the natural identifications $\Delta_{X_{2}}^{\text {Lie }} \stackrel{\sim}{\rightarrow} \breve{\Delta}_{X_{2}}^{\text {Lie }}, \Delta_{Y_{2}}^{\text {Lie }} \rightarrow \breve{\Delta}_{Y_{2}}^{\text {Lie }}$ without $G_{K}$-actions.

(2) If we denote by $\alpha_{2}^{\Pi^{\mathrm{Lie}}}: \Pi_{X_{2}}^{\mathrm{Lie}} \stackrel{\sim}{\rightarrow} \Pi_{Y_{2}}^{\mathrm{Lie}}, \breve{\alpha}_{2}^{\Pi^{\mathrm{Lie}}}: \breve{\Pi}_{X_{2}}^{\mathrm{Lie}} \stackrel{\sim}{\rightarrow} \breve{\Pi}_{Y_{2}}^{\mathrm{Lie}}$ the semidirect products of $\alpha_{2}^{\Delta^{\mathrm{Lie}}}$ with the isomorphism $G_{K} \stackrel{\sim}{\rightarrow} G_{L}$ (i.e., determined by $\alpha$ ) relative to the respective actions of $G_{K}$ and $G_{L}$, then these morphisms make the following diagrams commute:

$$
\begin{aligned}
& \Pi_{\bar{X}_{x}^{\log }} \stackrel{\mathrm{Int}_{X}^{\Pi} \circ i_{1}^{\Pi}}{\longrightarrow} \Pi_{X_{2}}^{\mathrm{Lie}} \longrightarrow \Pi_{X^{\times 2}} \quad \Pi_{X_{2}}^{\mathrm{Lie}} \stackrel{s_{X}^{\Pi^{\mathrm{Lie}}}}{\longrightarrow} \breve{\Pi}_{X_{2}}^{\mathrm{Lie}} \\
& \alpha \downarrow \imath \quad \alpha_{2}^{\Pi^{\mathrm{Lie}}} \downarrow^{2} \quad \bar{\alpha} \times \bar{\alpha} \downarrow^{2} \quad \alpha_{2}^{\Pi^{\mathrm{Lie}}} \downarrow \quad \breve{\alpha}_{2}^{\Pi^{\mathrm{Lie}}} \downarrow \\
& \Pi_{\bar{Y}_{y}^{\log }} \stackrel{\operatorname{Int}_{Y}^{\Pi} \circ i_{1}^{\Pi}}{\longrightarrow} \Pi_{Y_{2}}^{\mathrm{Lie}} \longrightarrow \Pi_{Y \times 2} \quad \Pi_{Y_{2}}^{\mathrm{Lie}} \stackrel{s_{Y}^{\Pi^{\mathrm{Lie}}}}{\longrightarrow} \breve{\Pi}_{Y_{2}}^{\mathrm{Lie}}
\end{aligned}
$$

Proof

Assertion (a) follows immediately by transport of structure. Next, we consider assertion (b). Since $\alpha$ is assumed to be Frobenius-preserving, it follows from [13, Corollary 2.7(i)] that $\left(g_{X}, r_{X}\right)=\left(g_{Y}, r_{Y}\right)$ and that $\alpha$ induces an isomorphism $\alpha^{\mathrm{cpt}}: \Delta_{\bar{X}}^{\mathrm{ab}} \stackrel{\sim}{\rightarrow} \Delta_{\bar{Y}}^{\mathrm{ab}}$ and a bijective correspondence between the respective sets of cusps of $\bar{X}_{x}^{\log }, \bar{Y}_{y}^{\log }$ as well as isomorphisms of the inertia subgroups of cusps corresponding via this bijection. By applying these isomorphisms (together with the constructions of $\mathcal{L}_{X}^{1}, \mathcal{L}_{X}^{2}, \mathcal{L}_{Y}^{1}, \mathcal{L}_{Y}^{2}$ ), Lemma 5.6 yields $G_{K}$-equivariant isomor- 
phisms $\alpha^{\text {Lie }}: \operatorname{Lie}_{X_{2 / 1}}^{1} \cong \operatorname{Lie}_{Y_{2 / 1}}^{1}, \breve{\alpha}{ }^{\text {Lie }}: \breve{L i e}_{X_{2 / 1}}^{1} \cong \breve{L i e}_{Y_{2 / 1}}^{1}, \alpha_{2}^{\text {Lie }}: \operatorname{Lie}_{X_{2}} \cong \operatorname{Lie}_{Y_{2}}$, and $\breve{\alpha} \breve{L i e}_{2} \breve{L i e}_{X_{2}} \cong \breve{L i e}_{Y_{2}}$. These morphisms give rise to a $G_{K}$-equivariant commutative diagram as follows:

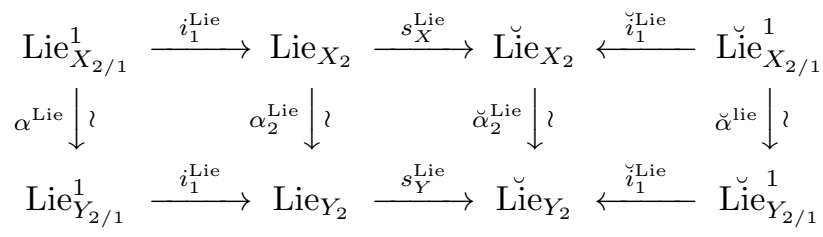

Then it follows from the functoriality of $\operatorname{Lin}(\cdot)$ that we obtain a $G_{K}$-equivariant commutative diagram as follows:

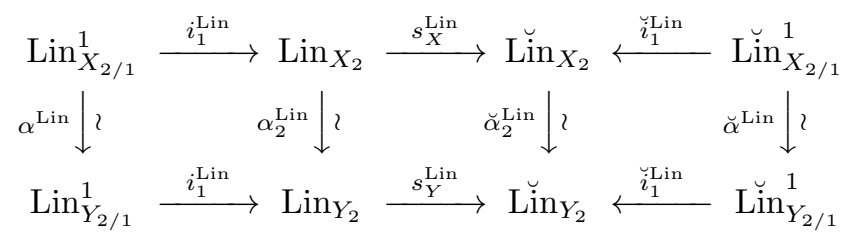

Note (see [12, Remark 35]) that modifying the choice $\left(C_{\sigma}^{X}\right)$ of a specific section $G_{K} \rightarrow D_{\tilde{x}}$ by a cocycle $G_{K} \rightarrow I_{\tilde{x}}$ determined by the choice $\left(C_{\delta}^{X}\right)$ affects the Galois invariant isomorphisms of Proposition 3.8(b) by conjugation by an element $c_{X}$ of the subgroup obtained by tensoring $I_{\tilde{x}}$ with $\mathbb{Q}_{l}$; a similar statement holds, with respect to some $c_{Y}$, for objects associated to $Y$ when we modify $\left(C_{\tau}^{Y}\right)$ by $\left(C_{\theta}^{Y}\right)$. One may verify easily that $\alpha$ maps $c_{X}$ to $c_{Y}$ and, hence, that $\alpha^{\text {Lin }}=$ $\breve{\alpha}^{\mathrm{Lin}}$ as a morphism of underlying topological groups (i.e., without $G_{K}$-actions). Next, recall that the morphisms $i_{1}^{\text {Lin }}$ and $s_{X}^{\text {Lin }} \circ i_{1}^{\text {Lin }}$ are compatible with the corresponding morphisms $i_{1}^{\mathrm{Lin}}$ and $s_{Y}^{\mathrm{Lin}} \circ i_{1}^{\mathrm{Lin}}$ associated to $Y$ via the natural identification of $\operatorname{Lin}_{X_{2}}$ with $\operatorname{Lin}_{X_{2}}$ (i.e., without $G_{K}$-actions). Also, let us recall that $\operatorname{Lin}_{X_{2}}$ (resp., $\breve{L i n}_{X_{2}}$ ) is generated by the images of $\operatorname{Lin}_{X_{2 / 1}}^{1} \stackrel{i_{1}^{\operatorname{Lin}} \rightarrow}{\rightarrow} \operatorname{Lin}_{X_{2}}$ (resp., $\breve{L i n}_{X_{2 / 1}}^{1} \stackrel{\breve{i}_{1}^{\text {Lin }}}{\rightarrow} \operatorname{Lin}_{X_{2}}$ ) and the composite $\operatorname{Lin}_{X_{2 / 1}}^{1} \stackrel{i_{1}^{\text {Lin }}}{\rightarrow} \operatorname{Lin}_{X_{2}} \stackrel{s_{X}^{\operatorname{Lin}}}{\rightarrow} \operatorname{Lin}_{X_{2}}=\operatorname{Lin}_{X_{2}}$ (resp. $\left.\breve{L i n}_{X_{2} / 1}^{1} \stackrel{\breve{i_{1}^{L i n}}}{\rightarrow} \breve{L i n}_{X_{2}}=\operatorname{Lin}_{X_{2}} \stackrel{s_{X}^{\text {Lin }}}{\rightarrow} \operatorname{Lin}_{X_{2}}\right)$. Since the restrictions of $\alpha_{2}^{\operatorname{Lin}}$ and $\breve{\alpha}_{2}^{\text {Lin }}$ to these image subgroups coincide by virtue of the equality $\alpha^{\text {Lin }}=\breve{\alpha}^{\text {Lin }}$, we obtain that $\alpha_{2}^{\operatorname{Lin}}=\breve{\alpha}_{2}^{\text {Lin }}$. Therefore, by construction, $\alpha_{2}^{\operatorname{Lin}}\left(=\breve{\alpha}_{2}^{\text {Lin }}\right)$ induces the required $G_{K}$-equivariant isomorphism $\alpha_{2}^{\Delta^{\text {Lie }}}: \Delta_{X_{2}}^{\text {Lie }} \stackrel{\sim}{\rightarrow} \Delta_{Y_{2}}^{\text {Lie }}$ of topological groups satisfying conditions (1) and (2). This completes the proof of assertion (b).

One of main results of this article, that is, a slightly generalized version of Theorem $\mathrm{A}$, is the following.

\section{THEOREM 5.9}

Let $X$ (resp., $Y$ ) be a hyperbolic curve over a finite field $K$ (resp., L), let $x$ be a $K$-rational point of $\bar{X}$ (resp., $y$ be an L-rational point of $\bar{Y}$ ), let $X_{2}$ (resp., $Y_{2}$ ) be the second configuration space associated to $X$ (resp., $Y$ ), let $\bar{X}_{x}^{\log }$ (resp., $\bar{Y}_{y}^{\log }$ ) be the cuspidalization of $X$ at $x$ (resp., of $Y$ at $y$; see Definition 4.2), and let 
$D_{\tilde{x}} \subseteq \Pi_{\bar{X}_{x}^{\log }}$ (resp., $D_{\tilde{y}} \subseteq \Pi_{\bar{Y}_{y}^{\log }}$ ) be a specific decomposition group of the diagonal cusp $\tilde{x}^{\log }$ (resp., $\tilde{y}^{\log }$; see the discussion following Remark 4.1.1). Let

$$
\alpha: \Pi_{\bar{X}_{x}^{\log }} \stackrel{\sim}{\longrightarrow} \Pi_{\bar{Y}_{y}^{\log }}
$$

be a Frobenius-preserving isomorphism (see Definition 5.7(a)) which maps $D_{\tilde{x}}$ onto $D_{\tilde{y}}$. Let us denote by $\bar{\alpha}: \Pi_{X} \stackrel{\sim}{\rightarrow} \Pi_{Y}$ the isomorphism obtained by passing to the quotients $\Pi_{\bar{X}_{x}^{\log }} \rightarrow \Pi_{X}, \Pi_{\bar{Y}_{y}^{\log }} \rightarrow \Pi_{Y}$. Let us denote by $D_{x} \subseteq \Pi_{X}$ (resp., $D_{y} \subseteq \Pi_{Y}$ ) the decomposition group of $x$ (resp., the decomposition group of $y$ ) determined by the image of $D_{\tilde{x}}$ in $\Pi_{X}$ (resp., as the image of $D_{\tilde{y}}$ in $\Pi_{Y}$ ) via the

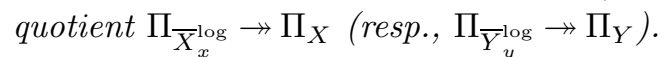

Then there exists an isomorphism

$$
\alpha_{2}: \Pi_{X_{2}} \stackrel{\sim}{\longrightarrow} \Pi_{Y_{2}}
$$

which is uniquely determined up to composition with an inner automorphism (of either the domain or codomain) by the condition that it is compatible with the natural switching automorphisms (see the discussion following Remark 4.1.1) and with the specific decomposition groups associated to the respective diagonal divisors determined by $D_{\tilde{x}}, D_{\tilde{y}}$ (see Lemma 4.4(b)), which fits into the following commutative square:

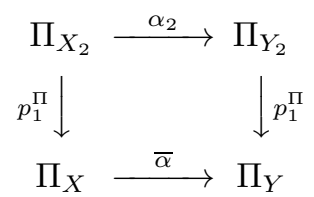

and induces $\alpha$ upon restriction to the inverse images (via the vertical arrows of (**)) of $D_{x} \subseteq \Pi_{X}$ and $D_{y} \subseteq \Pi_{Y}$.

Proof

Let us fix specific choices of $\left(C_{i_{1}}^{X}\right),\left(C_{p_{1}}^{X}\right),\left(C_{i_{1}}^{Y}\right),\left(C_{p_{1}}^{Y}\right)$. By applying Proposition 5.8 to these choices and the choices of $\left(C_{\tilde{x}}^{X}\right),\left(C_{\tilde{y}}^{Y}\right),\left(C_{x, y}^{\bar{X}_{x}^{\log } \sim \bar{Y}_{y}^{\log }}\right)$ given by hypothesis, we obtain a commutative diagram as follows:

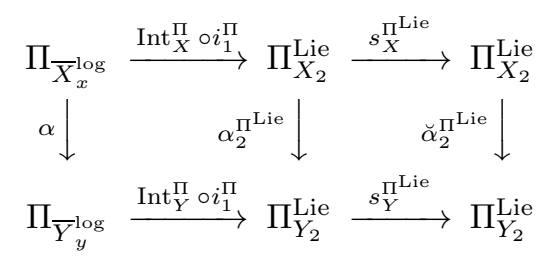

Now observe that, by the various constructions involved, $s_{X}^{\Pi^{\mathrm{Lie}}} \circ s_{X}^{\Pi^{\mathrm{Lie}}}=\mathrm{id}_{\Pi_{X_{2}}^{\mathrm{Lie}}}$, and $s_{X}^{\Pi^{\mathrm{Lie}}} \circ \operatorname{Int}_{X}^{\Pi} \circ i_{1}^{\Pi}$ coincides with $\operatorname{Int}_{X}^{\Pi} \circ i_{2}^{\Pi}$ for some $i_{2}^{\Pi}: \Pi_{\bar{X}_{x}^{\log }} \rightarrow \Pi_{\bar{X}_{2}^{\log }}$ (within the conjugacy class of homomorphisms determined by $\left.i_{2}^{\Pi}\right)$ induced by $i_{2}: \bar{X}_{x}^{\log } \rightarrow$ $\bar{X}_{2}^{\log }$. Thus, it follows from Proposition 3.4(b) that $\left(\operatorname{Int}_{X}^{\Pi} \circ i_{1}^{\Pi}\right)\left(\Pi_{\bar{X}_{x}^{\log }}\right)$ and $\left(s_{X}^{\Pi^{\mathrm{Lie}}} \circ\right.$ $\left.\operatorname{Int}_{X}^{\Pi} \circ i_{1}^{\Pi}\right)\left(\Delta_{X_{2 / 1}}^{1}\right)$ generate $\Pi_{X_{2}}$, and that $\Pi_{X_{2}}$ is preserved by the action of $s_{X}^{\Pi^{\mathrm{Lie}}}$. 
Similarly, $\Pi_{Y_{2}}$ is generated by $\left(\operatorname{Int}_{Y}^{\Pi} \circ i_{1}^{\Pi}\right)\left(\Pi_{\bar{Y}_{y}^{\log }}\right)$ and $\left(s_{Y}^{\Pi^{\mathrm{Lie}}} \circ \operatorname{Int}_{Y}^{\Pi} \circ i_{1}^{\Pi}\right)\left(\Delta_{Y_{2 / 1}}^{1}\right)$, and $\Pi_{Y_{2}}$ is preserved by the action of $s_{Y}^{\Pi^{\mathrm{Lie}}}$. Therefore, since the above diagram is commutative, $\alpha_{2}^{\Pi^{\mathrm{Lie}}}$ maps $\Pi_{X_{2}}$ onto $\Pi_{Y_{2}}$. Thus, the restriction $\alpha_{2}$ of $\alpha_{2}^{\Pi^{\mathrm{Lie}}}$ to $\Pi_{X_{2}}$ makes the diagram $(* *)$ commute and is compatible with the switching automorphisms. Since the specific inertia subgroup of $\Pi_{X_{2}}$ associated to the diagonal divisor determined by $D_{\tilde{x}}$ is the image of $I_{\tilde{x}} \subseteq \Pi_{\bar{X}_{x}^{\log }}$ via $\operatorname{Int}_{X}^{\Pi} \circ i_{1}^{\Pi}$ (see Lemma 4.4(b)), the isomorphism $\alpha_{2}$, which is an extension of the isomorphism $\alpha$, is compatible with the corresponding specific decomposition groups associated to the respective diagonal divisors. This completes the proof of the existence assertion.

Next, we consider uniqueness. Let $\dot{\alpha}_{2}, \ddot{\alpha}_{2}: \Pi_{X_{2}} \stackrel{\sim}{\rightarrow} \Pi_{Y_{2}}$ be isomorphisms, both of which make the diagram (**) commute and induce $\left.\alpha\right|_{\Delta_{X_{2 / 1}}^{1}}$ (i.e., the restriction of $\alpha$ to $\left.\Delta_{X_{2 / 1}}^{1}\right)$ upon restriction to the kernels of the vertical arrows of (**). Then $\dot{\alpha}_{2}^{-1} \circ \ddot{\alpha}_{2}$ determines an automorphism of the exact sequence

$$
1 \longrightarrow \Delta_{X_{2 / 1}}^{1} \stackrel{i_{1}^{\Pi}}{\longrightarrow} \Pi_{X_{2}} \stackrel{p_{1}^{\Pi}}{\longrightarrow} \Pi_{X} \longrightarrow 1,
$$

which induces the identity automorphisms on $\Delta_{X_{2 / 1}}^{1}$ and $\Pi_{X}$. This implies that $\dot{\alpha}_{2}^{-1} \circ \ddot{\alpha}_{2}$ is the identity morphism (see the last paragraph concerning topological groups in the Introduction).

COROLLARY 5.10

Let $X$ (resp., $Y$ ) be a hyperbolic curve over a finite field $K$ (resp., L), and let $x$, $x^{\prime}$ be $K$-rational points of $\bar{X}$ (resp., $y, y^{\prime}$ be L-rational points of $\left.\bar{Y}\right)$. Let

$$
\alpha: \Pi_{\bar{X}_{x}^{\log }} \longrightarrow \Pi_{\bar{Y}_{y}^{\log }}
$$

be a Frobenius-preserving isomorphism such that the decomposition groups of $\tilde{x}$ and $\tilde{y}$ (which are well defined up to conjugacy) correspond via $\alpha$. Suppose that the isomorphism $\bar{\alpha}: \Pi_{X} \stackrel{\sim}{\rightarrow} \Pi_{Y}$ induced by passing to the quotients $\Pi_{\bar{X}_{x}^{\log }} \rightarrow \Pi_{X}$, $\Pi_{\bar{Y}_{y}^{\log }} \rightarrow \Pi_{Y}$ maps the conjugacy class of the decomposition group of $x^{\prime}$ to the conjugacy class of the decomposition group of $y^{\prime}$. Then there exists a Frobeniuspreserving isomorphism

$$
\alpha^{\prime}: \Pi_{\bar{X}_{x^{\prime}}^{\log }} \longrightarrow \Pi_{\bar{Y}_{y^{\prime}}^{\log }}
$$

which is uniquely determined up to composition with an inner automorphism (of either the domain or codomain) by the condition that it induces $\bar{\alpha}$ upon passing to the respective quotients and maps the conjugacy class of the decomposition group of the diagonal cusp $\tilde{x^{\prime}}$ to the conjugacy class of the decomposition group of the diagonal cusp $\tilde{y}^{\prime}$.

Proof

The existence assertion follows from Theorem 5.9 and the fact that if $D_{x^{\prime}} \subseteq \Pi_{X}$, $D_{y^{\prime}} \subseteq \Pi_{Y}$ denote the decomposition groups of $x^{\prime}, y^{\prime}$, respectively, then we have natural isomorphisms $\Pi_{\bar{X}_{x^{\prime}}^{\log }} \cong D_{x^{\prime}} \times_{\Pi_{X}} \Pi_{X_{2}}, \Pi_{\bar{Y}_{y^{\prime}}^{\log }} \cong D_{y^{\prime}} \times_{\Pi_{Y}} \Pi_{Y_{2}}$. 
Next, we consider the uniqueness assertion. Let $\dot{\alpha}^{\prime}, \ddot{\alpha}^{\prime}: \Pi_{\bar{X}_{x^{\prime}}^{\log }} \stackrel{\sim}{\rightarrow} \Pi_{\bar{Y}_{y^{\prime}}^{\log }}$ be Frobenius-preserving isomorphisms, both of which induce $\bar{\alpha}$ upon passing to the respective quotients and map some specific decomposition group of the diagonal cusp $\tilde{x}^{\prime}$ to the same decomposition group of the diagonal cusp $\tilde{y}^{\prime}$. Write $\beta:=\left(\dot{\alpha}^{\prime}\right)^{-1} \circ \ddot{\alpha}^{\prime} \in \operatorname{Aut}\left(\Pi_{\bar{X}_{x^{\prime}} \log }\right)$. Then it follows from the existence portion of Theorem 5.9 that $\beta$ induces an element $\beta_{2} \in \operatorname{Aut}\left(\Pi_{X_{2}}\right)$ which induces the identity morphism of $\Pi_{X^{\times 2}}$ upon passing to the natural quotient $\Pi_{X_{2}} \rightarrow \Pi_{X^{\times 2}}$. Note that $\beta_{2}$ defines an element $\left[\beta_{2}\right] \in \mathrm{Out}^{\mathrm{FC}}\left(\Delta_{X_{2}}\right)$. Moreover, since $\beta_{2}$ induces the identity morphism of $\Pi_{X \times 2}$, it follows that $\left[\beta_{2}\right]$ maps to the identity element of $\operatorname{Out}\left(\Delta_{X}\right)$ (see [5] for the definition of and results concerning Out ${ }^{\mathrm{FC}}$ ). But $\operatorname{Out}^{\mathrm{FC}}\left(\Delta_{X_{2}}\right) \rightarrow \operatorname{Out}\left(\Delta_{X}\right)$ is injective (see, e.g., [5, Theorem A]), so we have $\left[\beta_{2}\right]=1$; that is, the restriction of $\beta_{2}$ to $\Delta_{X_{2}}$ coincides with an inner automorphism $\operatorname{Inn}(b)$ determined by an element $b$ of $\Delta_{X_{2}}$. By the construction of $\beta_{2}$, $\operatorname{Inn}(b)$ (preserves the subgroup $\Delta_{X_{2 / 1}}^{1}$ of $\Delta_{X_{2}}$ and) induces the identity morphism of $\Delta_{X}$ upon passing to the quotient $\Delta_{X_{2}} \rightarrow \Delta_{X_{2}} / \Delta_{X_{2 / 1}}^{1} \cong \Delta_{X}$. Since $\Delta_{X}$ is center-free (see Proposition 3.4(c)), we thus conclude that $b$ maps to the identity element of $\Delta_{X}$ via $\Delta_{X_{2}} \rightarrow \Delta_{X}$. In particular, $b$ is an element of $\Pi_{\bar{X}_{x^{\prime}}^{\log }}$. Thus, we have two automorphisms $\beta, \operatorname{Inn}(b)$ on $\Pi_{\bar{X}_{x^{\prime}}^{\log }}$ which coincide upon passing to the quotient $\Pi_{\bar{X}_{x^{\prime}} \log } \rightarrow D_{x^{\prime}} \subseteq \Pi_{X}$ as well as upon the restriction to $\Delta_{X_{2 / 1}}^{1} \subseteq \Pi_{\bar{X}_{x^{\prime}}^{\log }}$. This implies that $\beta=\operatorname{Inn}(b)$ (see the last paragraph concerning topological groups in the Introduction) and, hence, completes the proof of the uniqueness assertion.

REMARK 5.10.1

Any Frobenius-preserving isomorphism is quasi-point-theoretic (see [20, Corollary 2.10, Proposition 3.8], [13, Remark 10(iii)]), that is, induces a bijection between the sets of decomposition groups of the points of $\bar{X}, \bar{Y}$. Therefore, in the statement of Corollary 5.10, given a closed point $x^{\prime \prime}$ of $\bar{X}$, there always exists a closed point $y^{\prime \prime}$ of $\bar{Y}$ which corresponds, at the level of conjugacy classes of decomposition groups, to $x^{\prime \prime}$ via $\bar{\alpha}$ (but this choice is not necessarily unique).

\section{Cuspidalization problems for hyperbolic curves}

In this last section, we apply Theorem 5.9 to obtain group-theoretic constructions of the cuspidalization of a hyperbolic curve at a point infinitesimally close to a cusp (see Theorem 6.3) as well as of arithmetic fundamental groups of configuration spaces of arbitrary dimension (see Theorem 6.4).

We maintain the notation and setup of the discussion at the beginning of Section 5. Moreover, until the end of Theorem 6.3, we shall assume that $X$ is affine (i.e., $r>0$ ), and that $x$ is a split cusp of $X$, that is, $x \in \bar{X}(K) \backslash X(K)$. As discussed following Remark 4.1.1, the major and minor cuspidal components $\bar{X}^{\log ^{\prime}}, \overline{\mathbb{P}}_{X}^{\log ^{\prime}}$ at $x$, together with the nexus $\nu_{x}^{\log }$ at $x$, determine strict (see [6, Section 1.2]) closed sub-log schemes of $\bar{X}_{x}^{\log }$. These closed sub-log schemes determine 
subgroups well defined up to conjugacy

$$
\Pi_{\bar{X}^{\log ^{\prime}}}, \quad \Pi_{\bar{P}_{X}^{\log ^{\prime}}}, \quad \Pi_{\nu_{x}^{\log }} \subseteq \Pi_{\bar{X}_{x}^{\log }}
$$

which we shall refer to, respectively, as the major verticial, minor verticial, and nexus subgroups (see [14, Definition 1.4]).

LEMMA 6.1

Write

$$
D_{x}:=\operatorname{Im}\left(\Pi_{\bar{X}_{x}^{\log }} \stackrel{p_{1}^{\Pi} \circ i_{1}^{\Pi}}{\rightarrow} \Pi_{X}\right) .
$$

(Thus, $D_{x} \subseteq \Pi_{X}$ is a specific decomposition group of $x$, that is, well defined without any conjugacy indeterminacies.)

(a) For any choice of a specific major verticial subgroup $\Pi_{\bar{X}^{\log ^{\prime}}} \subseteq \Pi_{\bar{X}_{x}^{\log }}$, the composite morphism

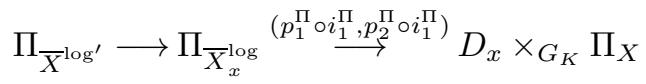

is an isomorphism. (In particular, the major verticial subgroups may be thought

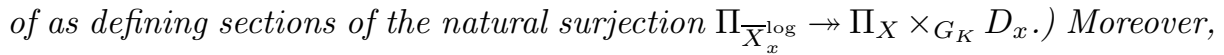
the inverse of this isomorphism maps the subgroup $D_{x} \times_{G_{K}} D_{x} \subseteq D_{x} \times_{G_{K}} \Pi_{X}$ to the nexus subgroup $\Pi_{\nu_{x}^{\log }} \subseteq \Pi_{\bar{X}^{\log { }^{\prime}}}$.

(b) In a similar vein, let $\overline{\mathbb{P}}_{K}^{\log }$ be the first log configuration space associated to a tripod $\mathbb{P}_{K}$ over $K$ (see Definition 3.1(b)). Then for any choice of a specific minor verticial subgroup $\Pi_{\overline{\mathbb{P}}_{X}^{\log }} \subseteq \Pi_{\bar{X}_{x}^{\log }}$, the composite morphism

$$
\Pi_{\overline{\mathbb{P}}_{X}^{\log { }^{\prime}}} \longrightarrow \Pi_{\bar{X}_{x}^{\log }} \stackrel{\left(p_{\mathbb{P}}^{\Pi}, p_{1}^{\Pi} \circ i_{1}^{\Pi}\right)}{\longrightarrow} \Pi_{\overline{\mathbb{P}}_{K}^{\log }} \times_{G_{K}} D_{x}
$$

- where $p_{\mathbb{P}}^{\Pi}$ denotes the homomorphism $\Pi_{\bar{X}_{x}^{\log }} \rightarrow \Pi_{\overline{\mathbb{P}}_{K}^{\log }}$ (well defined up to conjugation) induced by the natural morphism $\bar{X}_{x}^{\log } \rightarrow \overline{\mathbb{P}}_{K}^{\log }$ given by contracting $\bar{X}$ $\left(\subseteq \bar{X}_{x}\right)$ to $\nu_{x}-i s$ an isomorphism.

Proof

We shall only consider assertion (a), since assertion (b) follows from a similar argument. Let us consider the commutative diagram of natural morphisms of log schemes

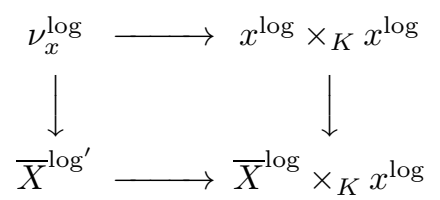

where the horizontal arrows are the strict closed immersions. Now recall that (1) két coverings may be constructed by means of descent with respect to (nonlogarithmic) étale morphisms; (2) restriction from a Henselian trait to its closed point induces an equivalence between the respective categories of két coverings 
(see [6]). Since the bottom horizontal arrow $\bar{X}^{\log ^{\prime}} \rightarrow \bar{X}^{\log } \times_{K} x^{\log }$ in the above diagram is an isomorphism on the respective complements of the images of the horizontal arrows in the above diagram, it suffices (by (1) and (2)) to verify that the induced morphism between the log inertia groups of $\nu_{x}^{\log }$ and $x^{\log } \times_{K} x^{\log }$

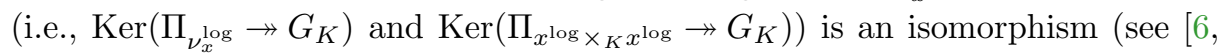
Section 4.7] for the terminology log inertia subgroup). Fix a chart, modeled on $\mathbb{N}$, of $x^{\log }$ (i.e., roots of a local uniformizer at $x$ in $\bar{X}$ ). Then such a chart determines charts, modeled on $\mathbb{N} \oplus \mathbb{N}$, of $x^{\log } \times_{K} x^{\log }$ and $\nu_{x}^{\log }$. By using these charts, one verifies easily that the homomorphism of monoids induced by the morphism $\nu_{x}^{\log } \rightarrow x^{\log } \times_{K} x^{\log }$ may be expressed as follows:

$$
\begin{aligned}
\mathbb{N} \oplus \mathbb{N} & \longrightarrow \mathbb{N} \oplus \mathbb{N}, \\
(a, b) & \mapsto(a+b, b) .
\end{aligned}
$$

Then, by applying the functor $\operatorname{Hom}\left((\cdot)^{\mathrm{gp}}, \mathbb{Z}_{l}(1)\right)$ to this morphism of monoids, one verifies immediately that the induced morphism of log inertia groups between $\nu_{x}^{\log }$ and $x^{\log } \times_{K} x^{\log }$ is an isomorphism.

LEMMA 6.2

Suppose that we fix a choice of a nexus subgroup $\Pi_{\nu_{x}^{\log }} \subseteq \Pi_{\bar{X}_{x}^{\log }}$ among its various $\Pi_{\bar{X}_{x}^{\log } \text {-conjugates. }}$

(a) There exists a unique pair of inclusions

$$
\Pi_{\bar{X}^{\log ^{\prime}}} \subseteq \Pi_{\bar{X}_{x}^{\log }}, \quad \Pi_{\overline{\mathbb{P}}_{X}^{\log }} \subseteq \Pi_{\bar{X}_{x}^{\log }}
$$

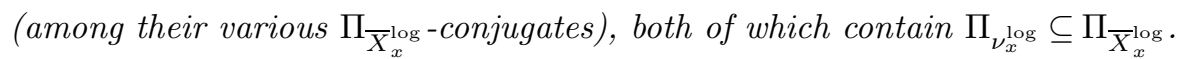

(b) The inclusions $\Pi_{\nu_{x}^{\log }} \subseteq \Pi_{\bar{X}^{\log }} \subseteq \Pi_{\bar{X}_{x}^{\log }}, \Pi_{\nu_{x}^{\log }} \subseteq \Pi_{\bar{P}_{X}^{\log }} \subseteq \Pi_{\bar{X}_{x}^{\log }}$ obtained in assertion (a) make the diagram

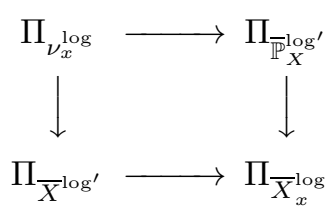

commute and co-Cartesian in the category of profinite groups equipped with an augmentation to $G_{K}$ whose kernel is pro-l.

Proof

Assertion (a) (resp., (b)) follows immediately from [14, Proposition 1.5(ii)] (resp., [14, Proposition 1.5(iii)]).

Next, we turn to the proof of Theorem B. Theorem 6.3 given below may be regarded as a slightly weakened version of Theorem B (as stated in the Introduction). This weakened version, however, will be sufficient to prove Theorem 6.4 below (which corresponds precisely to Theorem $\mathrm{C}$ in the Introduction). Moreover, one may obtain Theorem B (as stated in the Introduction) from Theorem C (see 
Remark 6.4.1). On the other hand, if we did not restrict our attention, in the statement of Theorem 6.3, to this slightly weakened version of Theorem B, then it would have been necessary to (essentially) repeat, in our proof of Theorem 6.4 below, arguments already applied in the proof of Theorem 6.3.

\section{THEOREM 6.3}

Let $X$ (resp., $Y)$ be an affine hyperbolic curve over a finite field $K$ (resp., L), and let $x$ (resp., $y$ ) be a K-rational (resp., L-rational) point of $\bar{X} \backslash X$ (resp., $\bar{Y} \backslash Y)$. Let

$$
\alpha: \Pi_{X} \stackrel{\sim}{\longrightarrow} \Pi_{Y}
$$

be a Frobenius-preserving isomorphism such that the decomposition groups of $x$ and $y$ (which are well defined up to conjugacy) correspond via $\alpha$. In the following, we shall apply the notational conventions introduced in the discussion following Remark 4.1.1.

Then there exist finite extensions $\dot{K}$ of $K$ and $\dot{L}$ of $L$ and an isomorphism

$$
\dot{\alpha}_{x, y}: \Pi_{\bar{X}_{x}^{\log }} \times_{G_{K}} G_{\dot{K}} \stackrel{\sim}{\longrightarrow} \Pi_{\bar{Y}_{y}^{\log }} \times_{G_{L}} G_{\dot{L}}
$$

which is uniquely determined up to composition with an inner automorphism (of either the domain or codomain) by the condition that it maps the conjugacy class of the decomposition group of $\tilde{x}$ to the conjugacy class of the decomposition group of $\tilde{y}$ and induces $\left.\alpha\right|_{\Pi_{X \times_{K} \dot{K}}}: \Pi_{X \times_{K} \dot{K}} \stackrel{\sim}{\rightarrow} \Pi_{Y \times_{L} \dot{L}}$ upon passing to the quotients

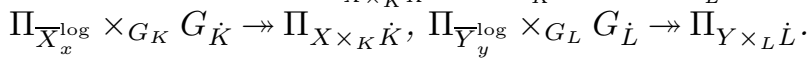

\section{Proof}

The asserted uniqueness follows immediately from the uniqueness portion of Corollary 5.10. Next, we shall consider the existence assertion. First, observe that there exists a connected finite étale covering $f: \dot{Z} \rightarrow X$, where $\dot{Z}$ is a hyperbolic curve over a finite extension field $\dot{K}$ of $K$ whose (smooth) compactification admits at least two distinct $\dot{K}$-rational points $z, z^{\prime}$ lying over $x$ at which $f$ is unramified. Indeed, this follows immediately from the well-known structure of $\Delta_{X}$. In the following, we shall, for simplicity, replace $\dot{K}$ by $K$ (i.e., assume that the base fields of $X$ and $\dot{Z}$ coincide).

Write $Z$ for the partial (smooth) compactification of $\dot{Z}$ at $z^{\prime}$ (i.e., a unique open subscheme $Z$ of the smooth compactification of $\dot{Z}$ containing $\dot{Z}$ and satisfying that $Z \backslash \dot{Z}=\left\{z^{\prime}\right\}$ ), and write $\bar{Z}_{z}^{\log }$ for the cuspidalization of $Z$ at $z$. Thus, the underlying scheme $\bar{Z}_{z}$ of $\bar{Z}_{z}^{\log }$ is proper. Denote by

$$
\bar{Z}^{\log ^{\prime}}, \quad \overline{\mathbb{P}}_{Z}^{\log ^{\prime}}, \quad \nu_{z}^{\log }
$$

the major and minor cuspidal components and the nexus of $\bar{Z}_{z}^{\log }$ at $z$, respectively (see the discussion at the beginning of Section 6). Let us fix specific choices of the decomposition groups $\dot{D}_{z} \subseteq \Pi_{\dot{Z}}$ of $z$ and $D_{x} \subseteq \Pi_{X}$ of $x$ such that $D_{x} \cap \Pi_{\dot{Z}}=\dot{D}_{z}$. Denote by $D_{z}$ the image of $\dot{D}_{z}$ via the quotient $\Pi_{\dot{Z}} \rightarrow \Pi_{Z}$ (which may be considered as the decomposition group of $z$ in $\Pi_{Z}$ ). Thus, the natural inclusion 
$\dot{D}_{z} \subseteq D_{x}$ is in fact an equality $\dot{D}_{z}=D_{x}$, and we have a natural isomorphism $\dot{D}_{z} \stackrel{\sim}{\rightarrow} D_{z}$. By applying Corollary 5.10 (see also Theorem 5.9) to the hyperbolic curve $\dot{Z}=Z \backslash\left\{z^{\prime}\right\}$ together with the $K$-rational points $z$ and $z^{\prime}$, we may reconstruct, group-theoretically from $\Pi_{\dot{Z}}$, the profinite group $\Pi_{\bar{Z}_{z}} \log$ together with its natural augmentation to $D_{z}$. Also, by [13, Corollary 2.7(iii)], we may reconstruct,

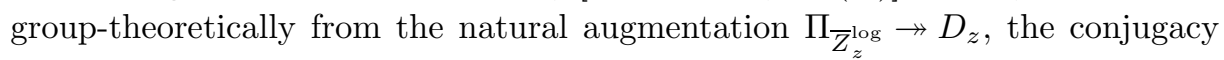
classes of the major verticial, minor verticial, and nexus subgroups of $\Pi_{\bar{Z}_{z}}$ log associated to the cuspidalization at $z$. Now let us fix specific choices of the major verticial, minor verticial, and nexus subgroups of $\Pi_{\bar{Z}_{z} \log }$

$$
\Pi_{\bar{Z}^{\log ^{\prime}}}, \quad \Pi_{\overline{\mathbb{P}}_{Z}^{\log { }^{\prime}}}, \quad \Pi_{\nu_{z}^{\log }}
$$

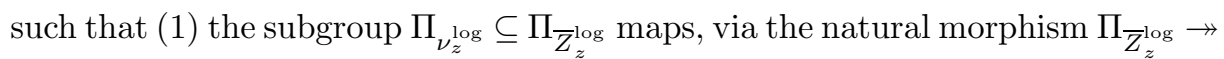
$\Pi_{Z}$, onto the subgroup $D_{z} ;$ and $(2) \Pi_{\nu_{z}^{\log }} \subseteq \Pi_{\bar{Z}^{\log }} \subseteq \Pi_{\bar{Z}_{z}^{\log }}$ and $\Pi_{\nu_{z}^{\log }} \subseteq \Pi_{\overline{\mathbb{P}}_{Z}^{\log ^{\prime}}} \subseteq$ $\Pi_{\bar{Z}_{z}^{\log }}$. (These choices are possible by virtue of Lemmas 6.1(a) and 6.2.) If we denote by $\overline{\mathbb{P}}_{K}^{\log }$ the first log configuration space associated to a tripod $\mathbb{P}_{K}$ over $K$, then we obtain (see Lemma 6.1(b)) a composite

$$
\Pi_{\nu_{z}^{\log }} \longrightarrow \Pi_{\overline{\mathbb{P}}_{Z}^{\log }} \stackrel{\sim}{\longrightarrow} \Pi_{\overline{\mathbb{P}}_{K}^{\log }} \times_{G_{K}} D_{x} .
$$

Here, we may regard $\Pi_{\bar{P}_{K}^{\log }}$ as an object group-theoretically reconstructed from $\Pi_{\overline{\mathbb{P}}_{Z}^{\log }}$ by thinking of $\Pi_{\overline{\mathbb{P}}_{K}^{\log }}$ as the quotient of the kernel of the natural composite augmentation $\Pi_{\overline{\mathbb{P}}_{Z}^{\log ^{\prime}}} \rightarrow D_{z} \rightarrow G_{K}$ (i.e., which is naturally isomorphic to $\Delta_{\mathbb{P}_{K}} \times$ $\mathbb{Z}_{l}(1)$ ) by its center (i.e., $\mathbb{Z}_{l}(1)$; see Proposition $3.4(\mathrm{c})$ ). Also, we obtain (see Lemma 6.1(a)) a diagram of natural morphisms

$$
D_{x} \times_{G_{K}} \Pi_{X} \longleftarrow \dot{D}_{z} \times_{G_{K}} \dot{D}_{z} \stackrel{\sim}{\longrightarrow} D_{z} \times_{G_{K}} D_{z} \stackrel{\sim}{\sim} \Pi_{\nu_{z}^{\text {log }}}
$$

induced, by restriction, from a diagram of natural morphisms

$$
D_{x} \times_{G_{K}} \Pi_{X} \hookleftarrow \dot{D}_{z} \times_{G_{K}} \Pi_{\dot{Z}} \rightarrow D_{z} \times_{G_{K}} \Pi_{Z} \tilde{\leftarrow} \Pi_{\bar{Z}^{\log ^{\prime}}}
$$

Thus, for suitable choices of the subgroups $\Pi_{\bar{X}^{\log ^{\prime}}}, \Pi_{\overline{\mathbb{P}}_{X}^{\log ^{\prime}}}, \Pi_{\nu_{x}^{\log }} \subseteq \Pi_{\bar{X}_{x}^{\log }}$ (see Lemma 6.2(a)), we obtain a natural commutative diagram

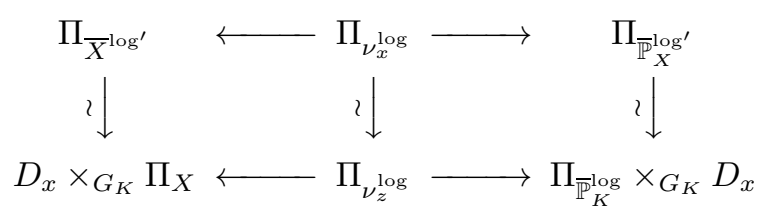

where the vertical arrows are all isomorphisms by Lemmas 6.1(a) and 6.1(b). In particular, it follows from Lemma $6.2(\mathrm{~b})$ that $\Pi_{\bar{X}_{x}^{\log }}$ may be identified with the colimit of the lower horizontal sequence - which, by the above discussion, may be reconstructed group-theoretically from the data $\left(\Pi_{X}, D_{x} \subseteq \Pi_{X}\right)$-in the above diagram. Therefore, by comparing this diagram to the corresponding diagram for $Y$, the proof is completed. 
Next, we consider Theorem C, that is, the cuspidalization problem for geometrically pro- $l$ fundamental groups of configuration spaces of (not necessarily proper) hyperbolic curves over finite fields.

THEOREM 6.4 ([12, THEOREM 3.1], [2, THEOREM 4.1])

Let $X$ (resp., $Y)$ be a hyperbolic curve over a finite field $K$ (resp., L). Let

$$
\alpha_{1}: \Pi_{X} \stackrel{\sim}{\longrightarrow} \Pi_{Y}
$$

be a Frobenius-preserving isomorphism. Then for any $n \in \mathbb{Z}_{\geq 0}$, there exists an isomorphism

$$
\alpha_{n}: \Pi_{X_{n}} \stackrel{\sim}{\longrightarrow} \Pi_{Y_{n}}
$$

which is uniquely determined up to composition with an inner automorphism (of either the domain or codomain) by the condition that it is compatible with the natural respective outer actions of the symmetric group on $n$ letters and makes the diagram

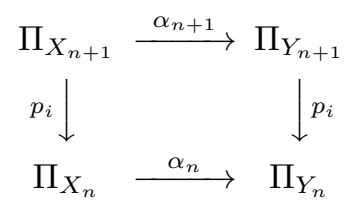

$(i=1, \ldots, n+1)$ commute

Proof

First, we recall that the case where $n=2$ and $X$ is proper follows from [12, Theorem 3.1]. Next, we consider the case where $n=2$ and $X$ is affine. As we noted in Definition 5.7(a), $\alpha_{1}$ induces an isomorphism

$$
\alpha_{0}: G_{K} \stackrel{\sim}{\rightarrow} G_{L}
$$

of profinite groups. Now, by combining Theorems 5.9 and 6.3 together with the fact that $\alpha_{1}$ is quasi-point-theoretic (see Remark 5.10.1), we conclude that $\alpha_{1}$ induces an isomorphism

$$
\dot{\alpha}_{2}: \Pi_{X_{2}} \times_{G_{K}} G_{\dot{K}} \stackrel{\sim}{\rightarrow} \Pi_{Y_{2}} \times_{G_{L}} G_{\dot{L}},
$$

where $G_{\dot{K}} \subseteq G_{K}, G_{\dot{L}} \subseteq G_{L}$ denote open subgroups corresponding to certain finite extensions $\dot{K}$ of $K$ and $\dot{L}$ of $L$, respectively. If we denote by $\alpha_{2}^{\Delta}$ the restriction of $\dot{\alpha}_{2}$ to $\Delta_{X_{2}}$, then (see Theorem 5.9) $\alpha_{2}^{\Delta}$ maps onto $\Delta_{Y_{2}}$, that is, determines an isomorphism

$$
\alpha_{2}^{\Delta}: \Delta_{X_{2}} \stackrel{\sim}{\rightarrow} \Delta_{Y_{2}}
$$

Let

$$
\gamma_{X}: G_{K} \rightarrow \operatorname{Out}^{\mathrm{FC}}\left(\Delta_{X_{2}}\right) \quad\left(\text { resp., } \gamma_{Y}: G_{L} \rightarrow \operatorname{Out}^{\mathrm{FC}}\left(\Delta_{Y_{2}}\right)\right)
$$

(see [5] for the definition and results concerning Out ${ }^{\mathrm{FC}}$ ) be the morphism obtained by lifting elements of $G_{K}$ (resp., $G_{L}$ ) via the surjection $\Pi_{X_{2}} \rightarrow G_{K}$ (resp., 
$\Pi_{Y_{2}} \rightarrow G_{L}$ ) and considering the action of these elements by conjugation. Then $\alpha_{2}^{\Delta}, \alpha_{0}$ give rise to two composites $\gamma_{Y} \circ \alpha_{0}$ and $\left[\alpha_{2}^{\Delta}\right] \circ \gamma_{X}$

$$
\gamma_{Y} \circ \alpha_{0},\left[\alpha_{2}^{\Delta}\right] \circ \gamma_{X}: G_{K} \longrightarrow \operatorname{Out}^{\mathrm{FC}}\left(\Delta_{Y_{2}}\right)
$$

where $\left[\alpha_{2}^{\Delta}\right]$ denotes the isomorphism $\mathrm{Out}^{\mathrm{FC}}\left(\Delta_{X_{2}}\right) \stackrel{\sim}{\rightarrow} \mathrm{Out}^{\mathrm{FC}}\left(\Delta_{Y_{2}}\right)$ that sends an element $g \in \operatorname{Aut}\left(\Delta_{X_{2}}\right)$ to $\alpha_{2}^{\Delta} \circ g \circ\left(\alpha_{2}^{\Delta}\right)^{-1} \in \operatorname{Aut}\left(\Delta_{Y_{2}}\right)$. It follows from the constructions of $\alpha_{0}, \alpha_{2}^{\Delta}$ that $\gamma_{Y} \circ \alpha_{0}$ and $\left[\alpha_{2}^{\Delta}\right] \circ \gamma_{X}$ coincide after composition with the natural morphism $\operatorname{Out}^{\mathrm{FC}}\left(\Delta_{Y_{2}}\right) \rightarrow \operatorname{Out}\left(\Delta_{Y}\right)$. On the other hand, since $\operatorname{Out}^{\mathrm{FC}}\left(\Delta_{Y_{2}}\right) \rightarrow \operatorname{Out}\left(\Delta_{Y}\right)$ is injective (see, e.g., [5, Theorem A]), we conclude that $\gamma_{Y} \circ \alpha_{0}=\left[\alpha_{2}^{\Delta}\right] \circ \gamma_{X}$. Therefore, by applying the natural isomorphisms $\Pi_{X_{2}} \cong \Delta_{X_{2}} \stackrel{\text { out }}{\rtimes} G_{K}$ and $\Pi_{Y_{2}} \cong \Delta_{Y_{2}} \stackrel{\text { out }}{\rtimes} G_{L}$, we obtain an isomorphism $\Pi_{X_{2}} \cong \Pi_{Y_{2}}$, which satisfies the required uniqueness and compatibility properties (see the construction of $\dot{\alpha}_{2}$; see also Theorem 5.9). This completes the proof of the assertion in the case where $n=2$ and $X$ is affine.

Finally, the assertion in the case $n \geq 3$ follows from an inductive argument on $n$ applied to an argument similar to that given in the above discussion. Indeed, consider the natural exact sequence

$$
1 \longrightarrow \Delta_{\left(X \times_{K} \bar{K} \backslash\{x\}\right)_{n-1}} \longrightarrow \Pi_{X_{n}} \stackrel{q_{j}^{\Pi}}{\longrightarrow} \Pi_{X} \longrightarrow 1
$$

(which induces an isomorphism $\Pi_{X_{n}} \cong \Delta_{\left(X \times_{K} \bar{K} \backslash\{x\}\right)_{n-1}} \stackrel{\text { out }}{\rtimes} \Pi_{X}$ ), where $x$ denotes a $\bar{K}$-rational point of $X$, and $q_{j}^{\Pi}$ denotes the morphism induced by the projection $X_{n} \rightarrow X$ to the $j$ th factor. Since the natural morphism

$$
\operatorname{Out}^{\mathrm{FC}}\left(\Delta_{\left.\left(X \times_{K} \bar{K} \backslash\{x\}\right)_{n-1}\right)}\right) \rightarrow \mathrm{Out}^{\mathrm{FC}}\left(\Delta_{\left(X \times_{K} \bar{K} \backslash\{x\}\right)_{n-2}}\right)
$$

is injective (see [5]), we may carry out a similar argument to the above discussion

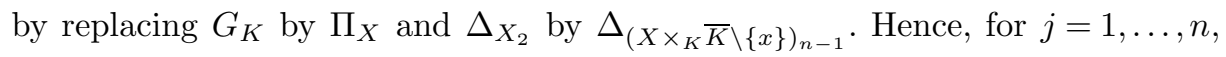
we obtain an isomorphism $\alpha_{n}^{j}: \Pi_{X_{n}} \stackrel{\sim}{\rightarrow} \Pi_{Y_{n}}$ that fits into a commutative diagram

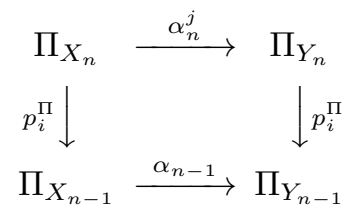

for $i=1, \ldots, n-1$. But it follows from the induction hypothesis (concerning the asserted uniqueness), together with the injectivity applied above, that the $\alpha_{n}^{j}$ 's coincide, for $j=1, \ldots, n$, up to composition with an inner automorphism, and that the asserted uniqueness and compatibility with symmetric group actions for $n$ are satisfied.

\section{REMARK 6.4.1}

As explained in the discussion preceding Theorem 6.3, one may obtain Theorem B (as stated in Introduction) directly from Theorem 6.4 as follows. Let $X, Y, x, y$, and $\alpha$ be as in the statement of Theorem B. Then, by applying Theorem 6.4 in 
the case $n=2$, we obtain a unique isomorphism

$$
\alpha_{2}: \Pi_{X_{2}} \stackrel{\sim}{\rightarrow} \Pi_{Y_{2}}
$$

that is well defined up to composition with an inner automorphism of either the domain or codomain, which fits into two commutative diagrams as follows:

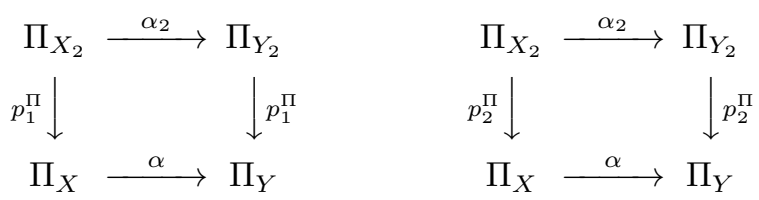

On the other hand, we may have natural identifications $\Pi_{\bar{X}_{x}^{\log }} \stackrel{\sim}{\rightarrow}\left(p_{2}^{\Pi}\right)^{-1}\left(D_{x}\right)$, $\Pi_{\bar{Y}_{y}^{\log }} \stackrel{\sim}{\rightarrow}\left(p_{2}^{\Pi}\right)^{-1}\left(D_{y}\right)$. Hence, the right-hand diagram above induces (since $\left.\alpha\left(\stackrel{y}{D}_{x}\right)=D_{y}\right)$ an isomorphism

$$
\alpha_{x, y}: \Pi_{\bar{X}_{x}^{\log }} \stackrel{\sim}{\rightarrow} \Pi_{\bar{Y}_{y}^{\log }}
$$

by restricting $\alpha_{2}$ to the inverse images (via the vertical arrows) of $D_{x} \subseteq \Pi_{X}$ and $D_{y} \subseteq \Pi_{Y}$. On the other hand, it follows from [13, Corollary 2.7(i)] that $\alpha_{x, y}$ maps the conjugacy class of the decomposition group of $\tilde{x}$ to the conjugacy class of the decomposition group of $\tilde{y}$. Thus, the left-hand commutative diagram above induces, by restricting the upper horizontal arrow of the diagram to the domain and codomain of $\alpha_{x, y}$, a commutative diagram $\left((*)^{\dagger}\right)$

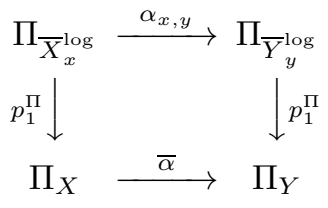

which completes the proof of Theorem B. (The proof of uniqueness is similar to the proof of the asserted uniqueness in Corollary 5.10.)

Finally, we conclude the article with the following corollary.

COROLLARY 6.5 ([2, COROLLARY 4.1])

Let $X$ (resp., $Y$ ) be a hyperbolic curve over a finite field $K$ (resp., L), and let $n \in \mathbb{Z}_{\geq 0}$. Let

$$
\alpha: \Pi_{X} \stackrel{\sim}{\longrightarrow} \Pi_{Y}
$$

be a Frobenius-preserving isomorphism, and let $x_{\bullet}:=\left\{x_{1}, \ldots, x_{n}\right\}$ be an ordered set of distinct $K$-rational points of $X$. Then there exist an ordered set $y_{\bullet}:=$ $\left\{y_{1}, \ldots, y_{n}\right\}$ of distinct $L$-rational points of $Y$ and an isomorphism

$$
\tilde{\alpha}: \Pi_{X \backslash\left\{x_{1}, \ldots, x_{n}\right\}} \stackrel{\sim}{\longrightarrow} \Pi_{Y \backslash\left\{y_{1}, \ldots, y_{n}\right\}}
$$

which is uniquely determined up to composition with an inner automorphism (of either the domain or codomain) by the condition that it induces $\alpha$ upon passing to quotients $\Pi_{X \backslash\left\{x_{1}, \ldots, x_{n}\right\}} \rightarrow \Pi_{X}, \Pi_{Y \backslash\left\{y_{1}, \ldots, y_{n}\right\}} \rightarrow \Pi_{Y}$ and maps the conjugacy 
classes of the decomposition groups of the points in $x$. to the conjugacy classes of the decomposition groups of the points in $y$. in the order of numbering.

Proof

The existence assertion follows, by induction on $n$, from Theorem 6.4 together with the fact that any Frobenius-preserving isomorphism between hyperbolic curves over finite fields preserves the set of decomposition groups of closed points (see Remark 5.10.1). The asserted uniqueness follows from the uniqueness asserted in Corollary 5.10 applied successively to the cuspidalizations at corresponding points of $x_{\bullet}$ and $y_{\bullet}$.

Acknowledgments. The author would like to express his sincere gratitude to Professors Shinichi Mochizuki and Yuichiro Hoshi for their warm encouragement, suggestions, and helpful advice, as well as to Professor Akio Tamagawa for constructive comments concerning this article. The author would like to thank the referee for carefully reading the manuscript and making some comments and suggestions.

\section{References}

[1] A. Grothendieck, Revêtements étale et groupe fondamental, Séminaire de Géométrie Algébrique du Bois-Marie 1960-61 (SGA 1), Lecture Notes in Math. 224, Springer, Berlin, 1971. MR 0354651.

[2] Y. Hoshi, Absolute anabelian cuspidalizations of configuration spaces of proper hyperbolic curves over finite fields, Publ. Res. Inst. Math. Sci. 45 (2009), 661-744. MR 2569565. DOI 10.2977/prims/1249478963.

[3] - The exactness of the log homotopy sequence, Hiroshima Math. J. 39 (2009), 61-121. MR 2499198.

[4] On the fundamental groups of log configuration schemes, Math. J. Okayama Univ. 51 (2009), 1-26. MR 2482403.

[5] Y. Hoshi and S. Mochizuki, On the combinatorial anabelian geometry of nodally nondegenerate outer representations, Hiroshima Math. J. 41 (2011), 275-342. MR 2895284.

[6] L. Illusie, "An overview of the work of K. Fujiwara, K. Kato and C. Nakayama on logarithmic étale cohomology" in Cohomologies p-adiques et applications arithmetiques, II, Astérisque 279, Soc. Math. France, Montrouge, 2002, 271-322. MR 1922832.

[7] K. Kato, "Logarithmic structures of Fontaine-Illusie" in Algebraic Analysis, Geometry, and Number Theory (Baltimore, Md., 1988), Johns Hopkins Univ. Press, Baltimore, 1989, 191-224. MR 1463703.

[8] F. F. Knudsen, The projectivity of the moduli space of stable curves, II: The stacks $M_{g, r}$, Math. Scand. 52 (1983), 161-199. MR 0702953. 
[9] S. Mochizuki, Extending families of curves over log regular schemes, J. Reine Angew. Math. 511 (1999), 43-71. MR 1695789. DOI 10.1515/crll.1999.511.43.

[10] , "The absolute anabelian geometry of hyperbolic curves" in Galois Theory and Modular Forms, Dev. Math. II, Kluwer Acad., Boston, 2004, 77-122. MR 2059759. DOI 10.1007/978-1-4613-0249-0_5.

[11] Galois sections in absolute anabelian geometry, Nagoya Math. J. 179 (2005), 17-45. MR 2164400.

[12] Absolute anabelian cuspidalizations of proper hyperbolic curves, Kyoto J. Math. 47 (2007), 451-539. MR 2402513.

[13] A combinatorial version of the Grothendieck conjecture, Tohoku Math. J. (2) 59 (2007), 455-479. MR 2365351.

[14] - On the combinatorial cuspidalization of hyperbolic curves, Osaka J. Math. 47 (2010), 651-715. MR 2768498.

[15] S. Mochizuki and A. Tamagawa, The algebraic and anabelian geometry of configuration spaces, Hokkaido Math. J. 37 (2008), 75-131. MR 2395079. DOI 10.14492/hokmj/1253539588.

[16] D. Mumford, Abelian Varieties, Tata Inst. Fund. Res. Stud. Math. 5, Oxford Univ. Press, London, 1970. MR 0282985.

[17] H. Nakamura, Galois rigidity of the étale fundamental groups of punctured projective lines, J. Reine Angew. Math. 411 (1990), 205-216. MR 1072981. DOI 10.1515/crll.1990.411.205.

[18] H. Nakamura, N. Takao, and R. Ueno, Some stability properties of Teichmüller modular function fields with pro-l weight structures, Math. Ann. 302 (1995), 197-213. MR 1336334. DOI 10.1007/BF01444493.

[19] J. Stix, A monodromy criterion for extending curves, Int. Math. Res. Not. IMRN 2005, no. 29, 1787-1802. MR 2172341. DOI 10.1155/IMRN.2005.1787.

[20] A. Tamagawa, The Grothendieck conjecture for affine curves, Compos. Math. 109 (1997), 135-194. MR 1478817. DOI 10.1023/A:1000114400142.

Graduate School of Mathematical Sciences, University of Tokyo, 3-8-1 Komaba, Meguro, Tokyo, 153-8914, Japan; wkbysh@ms.u-tokyo.ac.jp 